\title{
Wave Equation for Operators with Discrete Spectrum and Irregular Propagation Speed
}

\author{
Michael Ruzhansky(D) \& Niyaz Tokmagambetov
}

\author{
Communicated by G. DAL Maso
}

\begin{abstract}
Given a Hilbert space $\mathcal{H}$, we investigate the well-posedness of the Cauchy problem for the wave equation for operators with a discrete non-negative spectrum acting on $\mathcal{H}$. We consider the cases when the time-dependent propagation speed is regular, Hölder, and distributional. We also consider cases when it is strictly positive (strictly hyperbolic case) and when it is non-negative (weakly hyperbolic case). When the propagation speed is a distribution, we introduce the notion of "very weak solutions" to the Cauchy problem. We show that the Cauchy problem for the wave equation with the distributional coefficient has a unique "very weak solution" in an appropriate sense, which coincides with classical or distributional solutions when the latter exist. Examples include the harmonic and anharmonic oscillators, the Landau Hamiltonian on $\mathbb{R}^{n}$, uniformly elliptic operators of different orders on domains, Hörmander's sums of squares on compact Lie groups and compact manifolds, operators on manifolds with boundary, and many others.
\end{abstract}

\section{Contents}

1. Introduction . . . . . . . . . . . . . . . . . . . . 1162

2. Main Results, Part I . . . . . . . . . . . . . . . . . . . . . . . . . . . 1164

3. Examples . . . . . . . . . . . . . . . . . . . . . . . . 1167

3.1. Landau Hamiltonian in 2D . . . . . . . . . . . . . . . . . . . . . . 1168

3.2. Harmonic and Anharmonic Oscillators . . . . . . . . . . . . . . . . . . . 1169

3.3. Higher Dimensional Hamiltonian . . . . . . . . . . . . . . . . . . . . . . . 1170

3.4. Regular Elliptic Boundary Value Problems . . . . . . . . . . . . . . . . . . 1170

Michael Ruzhansky was supported in parts by the EPSRC Grant EP/K039407/1 and by the Leverhulme Grant RPG-2014-02. Niyaz Tokmagambetov was supported by the MESRK (Ministry of Education and Science of the Republic of Kazakhstan) Grant 0773/GF4. No new data was collected or generated during the course of research. 
3.5. Sums of Squares on Compact Lie Groups ～. . . . . . . . . . . . . . 1170

3.6. Weighted Sub-Laplacians and Sub-Riemannian Ornstein-Uhlenbeck Operators

on H-Type and Métivier Groups ． . . . . . . . . . . . . . . . . . . 1171

3.7. Operators on Manifolds with Boundary . . . . . . . . . . . . . . . . 1171

3.8. Differential Operators with Strong Regular Boundary Conditions . . . . . . 1172

4. Main Results, Part II: Very Weak Solutions . . . . . . . . . . . . . . . . . . 1173

5. $\mathcal{L}$-Fourier Analysis . . . . . . . . . . . . . . . . . . . . . . . . . 1177

6. Proofs of Part I: Theorems 2.1-2.4 f . . . . . . . . . . . . . . . . . . . 1183

6.1. Case I.1: Proof of Theorem $2.1 \ldots \ldots \ldots$

6.2. Case I.2: Proof of Theorem 2.2 (a) . . . . . . . . . . . . . . . . . . . . . 1186

6.3. Case I.2: Proof of Theorem 2.2 (b) . . . . . . . . . . . . . . . . . . . . . 1189

6.4. Case I.3: Proof of Theorem 2.3 (a) . . . . . . . . . . . . . . . . . . . . 1190

6.5. Case I.3: Proof of Theorem 2.3 (b) . . . . . . . . . . . . . . . . . . 1192

6.6. Case I.4: Proof of Theorem 2.4 (a) . . . . . . . . . . . . . . . . . . . . . . . . . . . . . . . 1193

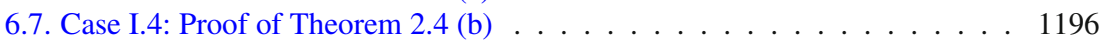

7. Proofs of Part II . . . . . . . . . . . . . . . . . . . . . . . 1196

7.1. Existence of Very Weak Solutions . . . . . . . . . . . . . . . . . 1196

7.2. Consistency with the Classical Well-Posedness . . . . . . . . . . . . . . . 1199

References . . . . . . . . . . . . . . . . . . . . . . . . . . . . . . . 1204

\section{Introduction}

Let $\mathcal{L}$ be a densely defined linear operator with the discrete spectrum

$$
\{\lambda \xi \geq 0: \xi \in \mathcal{I}\}
$$

on a separable Hilbert space $\mathcal{H}$. The main (and only) assumption in this paper will be that the system of eigenfunctions $\left\{e_{\xi}: \xi \in \mathcal{I}\right\}$ is a Riesz basis in $\mathcal{H}$, where $\mathcal{I}$ is a countable set. This means that every element of $\mathcal{H}$ has a unique decomposition with respect to this basis.

Note that we do not assume a-priori that the operator $\mathcal{L}$ is self-adjoint or normal in any sense so that the basis $\left\{e_{\xi}: \xi \in \mathcal{I}\right\}$ does not have to be orthogonal.

In this paper, for a non-negative function $a=a(t) \geq 0$ and for the source term $f=f(t) \in \mathcal{H}$, we are interested in the well-posedness of the Cauchy problem for the operator $\mathcal{L}$ with the propagation speed given by $a$ :

$$
\left\{\begin{aligned}
\partial_{t}^{2} u(t)+a(t) \mathcal{L} u(t) & =f(t), t \in[0, T], \\
u(0) & =u_{0} \in \mathcal{H}, \\
\partial_{t} u(0) & =u_{1} \in \mathcal{H} .
\end{aligned}\right.
$$

In order to provide a comprehensive analysis of such problems, we will treat several cases depending on the properties of $a$, and to a lesser extent of $f$. The reason behind this is that in each case the optimal results that we can get are different and depend on the properties of $a$. More specifically, we consider the following cases:

(I.1) The coefficient $a$ and the source term $f$ are regular enough: $a \in \operatorname{Lip}, f \in C$, and $a \geq a_{0}>0$. This is the classical case where we show the (natural) well-posedness of (1.1) in Sobolev spaces associated to $\mathcal{L}$. 
(I.2) We consider the case when $a \in C^{\alpha}, 0<\alpha<1, a \geq a_{0}>0$, is strictly positive and Hölder of order $\alpha$. In this case it is well-known already for $\mathcal{L} u=-u^{\prime \prime}$ on $\mathbb{R}$ that the Cauchy problem may be not well-posed in $C^{\infty}$ or in $\mathcal{D}^{\prime}$ (see e.g. Colombini, de Giorgi and Spagnolo [11] or [13]) and the Gevrey spaces appear naturally. Here we prove the well-posedness of (1.1) in the scale of $\mathcal{L}$-Gevrey spaces and $\mathcal{L}$-ultradistributions that we introduce for this purpose.

(I.3) We consider the case when $a \in C^{\ell}, \ell \geq 2, a \geq 0$, is regular but may be equal to zero (the weakly hyperbolic case). In this case there may be also no well-posedness in $C^{\infty}$ or in $\mathcal{D}^{\prime}$ already for $\mathcal{L} u=-u^{\prime \prime}$ on $\mathbb{R}$. Here we also prove the well-posedness of (1.1) in the scale of $\mathcal{L}$-Gevrey spaces and $\mathcal{L}$-ultradistributions.

(I.4) The last 'regular' case is the weakly hyperbolic case with Hölder propagation speed: when $a \in C^{\alpha}, 0<\alpha<2, a \geq 0$. Here we also prove the wellposedness of (1.1) in the scale of $\mathcal{L}$-Gevrey spaces and $\mathcal{L}$-ultradistributions depending on $\alpha$.

Consequently, we also consider the cases when $a$ is less regular than Hölder, allowing it to be a (positive) distribution, for example allowing the case

$$
a=1+\delta,
$$

involving the $\delta$-distribution. Such type of setting appears in applications, for example when one is looking at the behaviour of a particle in irregular electromagnetic fields: in this case $\mathcal{L}$ is the Landau Hamiltonian on $\mathbb{R}^{n}$, and the corresponding wave equation was analysed by the authors in [54]. While from the physical point of view (of irregular electromagnetic fields) such situation is natural and one expects the well-posedness, mathematically equation (1.1) is difficult to handle because of the general impossibility to multiply distributions (recall the famous Schwartz impossibility result from [58]).

With the setting of $\mathcal{L}$ being a second order invariant partial differential operator in $\mathbb{R}^{n}$, in [25], Claudia Garetto and the first-named author introduced the notion of "very weak solutions", proving their existence, uniqueness and consistency with classical or distributional solutions should the latter exist, for wave-type equations in $\mathbb{R}^{n}$. The setting of the present paper is different (since we assume that $\mathcal{L}$ has a discrete spectrum), and in [54] the authors proved the existence, uniqueness and consistency for the case when $\mathcal{L}$ is the Landau Hamiltonian on $\mathbb{R}^{n}$ (see the example in Sect. 3.1). Thus, the second aim of this paper is to develop the general notion of very weak solutions for the abstract problem (1.1). In a particular case of the Landau Hamiltonian, the results of this paper also extend those in [54] by allowing a wider class of admissible Cauchy data $u_{0}, u_{1}$. The analysis of very weak solutions is based on the results and techniques of cases (I.1)-(I.4). Thus, in this paper we also consider the following situations:

(II.1) The coefficient $a \geq a_{0}>0$ is a strictly positive distribution and the Cauchy data $u_{0}, u_{1}$ and the source term $f(t)$ belong to the $\mathcal{L}$-Sobolev spaces $H_{\mathcal{L}}^{s}$ for some $s \in \mathbb{R}$. In this case we prove the existence and uniqueness of Sobolevtype very weak solutions, and their consistency with cases (I.1)-(I.4) when we know that stronger solutions exist. 
(II.2) The coefficient $a \geq 0$ is a non-negative distribution and the Cauchy data $u_{0}, u_{1}$ and $f(t)$ are $\mathcal{L}$-distributions or $\mathcal{L}$-ultradistributions. In this case we prove the existence and uniqueness of ultradistributional-type very weak solutions, and their consistency with cases (I.2)-(I.4) when we know that ultradistributional solutions exist.

We divide the presentation of our results in two parts for the cases (I.1)-(I.5) and (II.1)-(II.2), respectively.

We note that we can partially remove the condition that the spectrum $\lambda \xi \geq 0$ is non-negative. Indeed, let $\mathcal{L}_{0}$ be a densely defined linear operator with the discrete spectrum $\left\{\lambda_{\xi} \in \mathbb{C}: \xi \in \mathcal{I}\right\}$ on the Hilbert space $\mathcal{H}$, and assume that the system of corresponding eigenfunctions $\left\{e_{\xi}: \xi \in \mathcal{I}\right\}$ is a Riesz basis in $\mathcal{H}$, where $\mathcal{I}$ is an ordered countable set. We denote by $\mathcal{L}:=\left|\mathcal{L}_{0}\right|$ the operator defined by assigning the eigenvalue $\left|\lambda_{\xi}\right|$ for each eigenfunction $e_{\xi}$. Moreover, if $\lambda \xi=0$ for some $\xi$, for example to define negative powers of an operator, we can put $\mathcal{L}:=\left|\mathcal{L}_{1}\right|$ to be the operator defined by the eigenvalue $\left(\left|\lambda_{\xi}\right|+c\right)$ to each eigenfunction $e_{\xi}$, with some positive $c>0$. We note that $\mathcal{L}$ is not the absolute value of $\mathcal{L}_{0}$ in the operator sense since $\mathcal{L}_{0}$ and its adjoint $\mathcal{L}_{0}^{*}$ may have different domains and are, in general, not composable. However, this is well-defined by the symbolic calculus developed in [53] (and extended in [55] to the full pseudo-differential calculus without the condition that eigenfunctions do not have zeros). Therefore, all the results of the paper extend to the Cauchy problem

$$
\left\{\begin{aligned}
\partial_{t}^{2} u(t)+a(t)\left|\mathcal{L}_{0}\right| u(t) & =f(t), t \in[0, T], \\
u(0) & =u_{0} \in \mathcal{H}, \\
\partial_{t} u(0) & =u_{1} \in \mathcal{H},
\end{aligned}\right.
$$

if we apply the results for (1.1) taking $\mathcal{L}=\left|\mathcal{L}_{0}\right|$ in the above sense.

Analogues of the considered problems for the wave equation for sub-Laplacians on the stratified groups and for Rockland operators on graded Lie groups have been considered in [56], but the situation there is different since the spectrum of the operator is continuous.

The organisation of the paper is as follows. In Sect. 2 we formulate the results for the cases (I.1)-(I.5). In Sect. 3 we give examples of different settings with different operators $\mathcal{L}$ satisfying our assumptions (discrete spectrum and a Riesz basis of eigenfunctions). In Sect. 4 we formulate the results for cases (II.1)-(II.2) corresponding to propagation speeds of low regularity. In Sect. 5 we review elements of the (nonharmonic) Fourier analysis associated to $\mathcal{L}$. In Sect. 6 we prove results of Part I from Sect. 2 and in Sect. 7 we prove results of Part II from Sect. 4.

\section{Main Results, Part I}

In our results below, concerning the Cauchy problem (1.1), we first carry out analysis in the strictly hyperbolic case $a(t) \geq a_{0}>0, a \in C^{1}([0, T])$. This is the 
regular strictly hyperbolic type case when we obtain the well-posedness in Sobolev spaces $H_{\mathcal{L}}^{s}$ associated to the operator $\mathcal{L}$ : for any $s \in \mathbb{R}$, we set

$$
H_{\mathcal{L}}^{s}:=\left\{f \in H_{\mathcal{L}}^{-\infty}: \mathcal{L}^{s / 2} f \in \mathcal{H}\right\}
$$

with the norm

$$
\|f\|_{H_{\mathcal{L}}^{s}}:=\left\|\mathcal{L}^{s / 2} f\right\|_{\mathcal{H}}
$$

The global space of $\mathcal{L}$-distributions $H_{\mathcal{L}}^{-\infty}$ is defined in Sect. 5. It is notationally more convenient to use the operator $\mathcal{L}^{1 / 2}$ in (2.1) because the operator in (1.1) is second order with respect to $t: \mathcal{L}$ is positive (that is, here, all eigenvalues are real and non-negative) so $\mathcal{L}^{1 / 2}$ is well defined by its spectral decomposition, but in Sect. 5 we will also make a symbol definition of $\mathcal{L}^{1 / 2}$. Namely, $\sigma_{\mathcal{L}^{1 / 2}}(\xi)=\lambda_{\xi}^{1 / 2}$. Anticipating the material of the next sections, using Plancherel's identity (5.9), in our case we can express the Sobolev norm as

$$
\|f\|_{H_{\mathcal{L}}^{s}}=\left(\sum_{\xi \in \mathcal{I}}\left|\lambda_{\xi}\right|^{s}\left(f, e_{\xi}^{*}\right)\left(e_{\xi}, f\right)\right)^{1 / 2} \simeq\left(\sum_{\xi \in \mathcal{I}}\left|\lambda_{\xi}\right|^{s}\left|\left(f, e_{\xi}\right)\right|^{2}\right)^{1 / 2},
$$

for any $s \in \mathbb{R}$, where $(\cdot, \cdot)$ is the inner product of $\mathcal{H}$, and $\left\{e_{\xi}^{*}\right\}_{\xi \in \mathcal{I}}$ is the biorthogonal basis to $\left\{e_{\xi}\right\}_{\xi \in \mathcal{I}}$. However, by Lemma 5.1 we can also use several equivalent expressions for the norm.

Moreover, if $\lambda_{\xi}=0$ for some $\xi$, in order to define negative powers of an operator, for eigenvectors corresponding to the zero eigenvalue, we can, without loss of generality, for some positive $c>0$, redefine $\mathcal{L}$ to be the operator assigning the eigenvalue $\left(\left|\lambda_{\xi}\right|+c\right)$ to each eigenfunction $e_{\xi}$.

Theorem 2.1. (Case I.1) Assume that $a \in \operatorname{Lip}([0, T])$ and that $a(t) \geq a_{0}>0$. For any $s \in \mathbb{R}$, if $f \in C\left([0, T], H_{\mathcal{L}}^{s}\right)$ and the Cauchy data satisfy $\left(u_{0}, u_{1}\right) \in H_{\mathcal{L}}^{s+1} \times$ $H_{\mathcal{L}}^{s}$, then the Cauchy problem (1.1) has a unique solution $u \in C\left([0, T], H_{\mathcal{L}}^{s+1}\right) \cap$ $C^{1}\left([0, T], H_{\mathcal{L}}^{s}\right)$ which satisfies the estimate

$$
\|u(t, \cdot)\|_{H_{\mathcal{L}}^{s+1}}^{2}+\left\|\partial_{t} u(t, \cdot)\right\|_{H_{\mathcal{L}}^{s}}^{2} \leq C\left(\left\|u_{0}\right\|_{H_{\mathcal{L}}^{s+1}}^{2}+\left\|u_{1}\right\|_{H_{\mathcal{L}}^{s}}^{2}+\|f\|_{C\left([0, T], H_{\mathcal{L}}^{s}\right)}^{2}\right) .
$$

As we have mentioned in the introduction, already in the setting of partial differential equations in $\mathbb{R}^{n}$, in the cases when $a$ is Hölder or non strictly positive, the well-posedness in the spaces of smooth functions or in the spaces of distributions fail. For example, it is possible to find smooth Cauchy data, taking also $f=0$, so that the Cauchy problem (1.1) would not have solutions in spaces of distributions, or a solution would exist but would not be unique - we refer to $[13,15]$ for respective examples. Therefore, already in such setting Gevrey spaces as well as spaces of ultradistributions appear naturally. Therefore, it is also natural to introduce these spaces in our setting. 
It will be convenient to also use the notation $H_{\mathcal{L}}^{\infty}$ for the space of test functions later on, defined by

$$
H_{\mathcal{L}}^{\infty}:=\bigcap_{s \geq 0} H_{\mathcal{L}}^{s} .
$$

Then we can define the $\mathcal{L}$-Gevrey (Roumieu) space $\gamma_{\mathcal{L}}^{s} \subset H_{\mathcal{L}}^{\infty}$ by the condition

$$
f \in \gamma_{\mathcal{L}}^{s} \Longleftrightarrow \exists A>0:\left\|\mathrm{e}^{A \mathcal{L}^{\frac{1}{2 s}}} f\right\|_{\mathcal{H}}<\infty,
$$

for $0<s<\infty$. The expression on the right hand side will be discussed in more detail in Sect. 5. Similarly, we can define the $\mathcal{L}$-Gevrey (Beurling) spaces by

$$
f \in \gamma_{\mathcal{L}}^{(s)} \Longleftrightarrow \forall A>0:\left\|\mathrm{e}^{A \mathcal{L}^{\frac{1}{2 s}}} f\right\|_{\mathcal{H}}<\infty,
$$

for $0<s<\infty$. These spaces are equipped with the corresponding inductive and projective topologies, see e.g. [22].

We denote by $H_{s}^{-\infty}$ and $H_{(s)}^{-\infty}$ the spaces of linear continuous functionals on $\gamma_{\mathcal{L}}^{s}$ and $\gamma_{\mathcal{L}}^{(s)}$, respectively. We call these the Gevrey Roumieu ultradistributions and the Gevrey Beurling ultradistributions, respectively. For further properties we refer to Sect. 5.

Theorem 2.2. (Case I.2) Assume that $a(t) \geq a_{0}>0$ and that $a \in C^{\alpha}([0, T])$ with $0<\alpha<1$. Then for initial data and for the source term

(a) $u_{0}, u_{1} \in \gamma_{\mathcal{L}}^{s}, f \in C\left([0, T] ; \gamma_{\mathcal{L}}^{s}\right)$,

(b) $u_{0}, u_{1} \in H_{(s)}^{-\infty}, f \in C\left([0, T] ; H_{(s)}^{-\infty}\right)$,

the Cauchy problem (1.1) has the unique solutions

(a) $u \in C^{2}\left([0, T] ; \gamma_{\mathcal{L}}^{s}\right)$,

(b) $u \in C^{2}\left([0, T] ; H_{(s)}^{-\infty}\right)$,

respectively, provided that

$$
1 \leq s<1+\frac{\alpha}{1-\alpha}
$$

We now consider the situation when the propagation speed $a(t)$ may become zero but is regular, i.e. $a \in C^{\ell}([0, T])$ for $\ell \geq 2$.

Theorem 2.3. (Case I.3) Assume that $a(t) \geq 0$ and that $a \in C^{\ell}([0, T])$ with $\ell \geq 2$. Then for initial data and for the source term

(a) $u_{0}, u_{1} \in \gamma_{\mathcal{L}}^{s}, f \in C\left([0, T] ; \gamma_{\mathcal{L}}^{s}\right)$,

(b) $u_{0}, u_{1} \in H_{(s)}^{-\infty}, f \in C\left([0, T] ; H_{(s)}^{-\infty}\right)$,

the Cauchy problem (1.1) has the unique solutions

(a) $u \in C^{2}\left([0, T] ; \gamma_{\mathcal{L}}^{s}\right)$,

(b) $u \in C^{2}\left([0, T] ; H_{(s)}^{-\infty}\right)$,

respectively, provided that 


$$
1 \leq s<1+\frac{\ell}{2}
$$

If a $(t) \geq 0$ belongs to $C^{\infty}([0, T])$ then the Cauchy problem (1.1) is well-posed as in (a) or (b) for every $s \geq 1$.

We now consider the case which is complementary to that in Theorem 2.3, namely, when the propagation speed $a(t)$ may become zero and is less regular, i.e. $a \in C^{\alpha}([0, T])$ for $0<\alpha<2$.

Theorem 2.4. (Case I.4) Assume that $a(t) \geq 0$ and that $a \in C^{\alpha}([0, T])$ with $0<\alpha<2$. Then, for initial data and for the source term

(a) $u_{0}, u_{1} \in \gamma_{\mathcal{L}}^{s}, f \in C\left([0, T] ; \gamma_{\mathcal{L}}^{s}\right)$,

(b) $u_{0}, u_{1} \in H_{(s)}^{-\infty}, f \in C\left([0, T] ; H_{(s)}^{-\infty}\right)$,

the Cauchy problem (1.1) has the unique solutions

(a) $u \in C^{2}\left([0, T] ; \gamma_{\mathcal{L}}^{s}\right)$,

(b) $u \in C^{2}\left([0, T] ; H_{(s)}^{-\infty}\right)$,

respectively, provided that

$$
1 \leq s<1+\frac{\alpha}{2}
$$

The proofs of the above theorems will be given in Sect. 6 .

Analogues of Parts (a) of the above theorems for the wave equation on $\mathbb{R}^{n}$ go back to Colombini, de Giorgi, and Spagnolo [11]. For higher order hyperbolic equations in $\mathbb{R}$ the Gevrey well-posedness was considered in [14] and [36] under assumptions corresponding to Cases I.2 (a) and I.3 (a), which were extended to $\mathbb{R}^{n}$ in [22] and [23], respectively. Other low regularity or multiple characteristics situations were considered in e.g. [7,10,12]. Equations with low regularity coefficients often come up in applications, see e.g. [29,30]. We refer to [22,23] for the history of the subject for hyperbolic equations on $\mathbb{R}^{n}$ with time-dependent coefficients, as well as for the sharpness of the orders from the theorems above in the case of $\mathbb{R}^{n}$. The mathematical analysis of hyperbolic equations with discontinuous coefficients goes back to Hurd and Sattinger [31]. We refer to [25] for the historical review of this topic.

\section{Examples}

In this section, as an illustration, we give several examples of the settings where our results are applicable. Of course, there are many other examples, here we collect the ones for which different types of partial differential equations have particular importance. We first discuss self-adjoint, and then non-self-adjoint operators. 


\subsection{Landau Hamiltonian in $2 D$}

First, we describe the setting of the Landau Hamiltonian in 2D. Here, the results of this paper partially recover and also extend the results obtained in [54]. More precisely, in [54] we considered the magnetic and electric fields of the operator separately, thus treating a more general model in the particular case of the Landau Hamiltonian. On the other hand, in [54] we obtained results corresponding to cases (I.1) and (II.1) only, not dealing with coefficients leading to the appearance of Gevrey type spaces. Therefore, the results of this paper extend those in [54] in the direction of Hölder propagation speeds as well as allowing more general Cauchy data and source terms.

We recall that the dynamics of a particle with charge $e$ and mass $m_{*}$ on the Euclidean $x y$-plane in the presence of the perpendicular constant homogeneous magnetic field is described by the Hamiltonian operator

$$
\mathcal{H}_{0}:=\frac{1}{2 m_{*}}\left(i h \nabla-\frac{e}{c} \mathbb{A}\right)^{2},
$$

where $h$ denotes Planck's constant, $c$ is the speed of light and $i$ the imaginary unit. In the sequel we can set $m_{*}=e=c=h=1$. With the symmetric gauge

$$
\mathbb{A}=-\frac{\mathrm{r}}{2} \times 2 \mathrm{~B}=(-B y, B x),
$$

where $\mathrm{r}=(x, y) \in \mathbb{R}^{2}$, and $2 B>0$ the strength of the magnetic field, one obtains the Landau Hamiltonian

$$
\mathcal{L}:=\frac{1}{2}\left(\left(i \frac{\partial}{\partial x}-B y\right)^{2}+\left(i \frac{\partial}{\partial y}+B x\right)^{2}\right),
$$

acting on the Hilbert space $L^{2}\left(\mathbb{R}^{2}\right)$. The spectrum of $\mathcal{L}$ consists of infinite number of eigenvalues (called the Euclidean Landau levels) with infinite multiplicity of the form

$$
\lambda_{n}=(2 n+1) B, \quad n=0,1,2, \ldots,
$$

see $[21,39]$. Denoting the eigenspace of $\mathcal{L}$ corresponding to the eigenvalue $\lambda_{n}$ by

$$
\mathcal{A}_{n}\left(\mathbb{R}^{2}\right)=\left\{\varphi \in L^{2}\left(\mathbb{R}^{2}\right), \mathcal{L} \varphi=\lambda_{n} \varphi\right\},
$$

its basis is given by (see $[2,32])$ :

$$
\left\{\begin{array}{l}
e_{k, n}^{1}(x, y)=\sqrt{\frac{n !}{(n-k) !}} B^{\frac{k+1}{2}} \exp \left(-\frac{B\left(x^{2}+y^{2}\right)}{2}\right)(x+i y)^{k} L_{n}^{(k)}\left(B\left(x^{2}+y^{2}\right)\right), \quad 0 \leq k, \\
e_{j, n}^{2}(x, y)=\sqrt{\frac{j !}{(j+n) !}} B^{\frac{n-1}{2}} \exp \left(-\frac{B\left(x^{2}+y^{2}\right)}{2}\right)(x-i y)^{n} L_{j}^{(n)}\left(B\left(x^{2}+y^{2}\right)\right), \quad 0 \leq j,
\end{array}\right.
$$

where $L_{n}^{(\alpha)}$ are the Laguerre polynomials given by

$$
L_{n}^{(\alpha)}(t)=\sum_{k=0}^{n}(-1)^{k} C_{n+\alpha}^{n-k} \frac{t^{k}}{k !}, \quad \alpha>-1 .
$$


This basis appears also in many related subjects, such as complex Hermite polynomials [34], in quantization [3,5,9], time-frequency analysis [1], partial differential equations [27], planar point processes [32], as well as in the Feynman-Schwinger displacement operator [46]. Their perturbations have been investigated in $[35,44]$, and the asymptotic behaviour of the eigenvalues was analysed in [37,40,41,48$50,57]$.

The results of this paper apply for the Cauchy problem (1.1) for the operator $\mathcal{L}$ from (3.2).

\subsection{Harmonic and Anharmonic Oscillators}

As a second example in any dimension $d \geq 1$, we consider the harmonic oscillator of Quantum Mechanics,

$$
\mathcal{L}:=-\Delta+|x|^{2}, \quad x \in \mathbb{R}^{d} .
$$

The operator $\mathcal{L}$ is essentially self-adjoint on $C_{0}^{\infty}\left(\mathbb{R}^{d}\right)$ with eigenvalues

$$
\lambda_{k}=\sum_{j=1}^{d}\left(2 k_{j}+1\right), \quad k=\left(k_{1}, \ldots, k_{d}\right) \in \mathbb{N}^{d},
$$

and with eigenfunctions

$$
u_{k}(x)=\prod_{j=1}^{d} P_{k_{j}}\left(x_{j}\right) \mathrm{e}^{-\frac{|x|^{2}}{2}}
$$

which form an orthogonal system in $L^{2}\left(\mathbb{R}^{d}\right)$. Here, $P_{n}(\cdot)$ is the $n$-th order Hermite polynomial, and

$$
P_{n}(t)=c_{n} \mathrm{e}^{\frac{|t|^{2}}{2}}\left(x-\frac{d}{d t}\right)^{n} \mathrm{e}^{-\frac{|t|^{2}}{2}}
$$

where $t \in \mathbb{R}$, and

$$
c_{n}=2^{-n / 2}(n !)^{-1 / 2} \pi^{-1 / 4} .
$$

For more details on the associated spectral analysis, see for instance [43].

Another family of examples is that of anharmonic oscillators, for example, of operators on $L^{2}(\mathbb{R})$ of the form

$$
\mathcal{L}:=-\frac{d^{2 k}}{d x^{2 k}}+x^{2 l}+p(x), \quad x \in \mathbb{R},
$$

for integers $k, l \geq 1$ and with $p(x)$ being a polynomial of degree $\leq 2 l-1$ with real coefficients. Such operators and their spectral properties have been analysed, for example, in [28]. 


\subsection{Higher Dimensional Hamiltonian}

Here we describe a higher dimensional example following [54]. Let $x=$ $\left(x_{1}, \ldots, x_{2 d}\right) \in \mathbb{R}^{2 d}$ and again setting all physical constants to be equal to 1 , in analogy to the case of $d=1$ in (3.2), let

$$
\mathcal{L}:=\frac{1}{2}(i \nabla-\mathbb{A})^{2},
$$

where

$$
\mathbb{A}=\left(-B_{1} x_{2}, B_{1} x_{1},-B_{2} x_{4}, B_{2} x_{3}, \ldots,-B_{d} x_{2 d}, B_{d} x_{2 d-1}\right),
$$

corresponding to the magnetic fields of constant strengths $2 B_{l}>0, l=1, \ldots, d$. The essentially self-adjoint operator $\mathcal{L}$ on $C_{0}^{\infty}\left(\mathbb{R}^{2 d}\right)$ in the Hilbert space

$$
L^{2}\left(\mathbb{R}^{2 d}\right)=\otimes_{1}^{d} L^{2}\left(\mathbb{R}^{2}\right)
$$

decomposes as

$$
\mathcal{L}=\mathcal{H}_{1} \otimes I^{\otimes(d-1)}+I \otimes \mathcal{H}_{2} \otimes I^{\otimes(d-2)}+\cdots+I^{\otimes(d-1)} \otimes \mathcal{H}_{d},
$$

with self-adjoint $2 \mathrm{D}$ operators $\mathcal{H}_{l}$ on $L^{2}\left(\mathbb{R}^{2}\right)$ as in (3.2). Let $k=\left(k_{1}, \ldots, k_{d}\right) \in \mathbb{N}_{0}^{d}$ be a multi-index. Then in analogy to (3.3), the spectrum of $\mathcal{L}$ consists of the infinitely degenerate eigenvalues

$$
\lambda_{k}=\sum_{l=1}^{d} B_{l}\left(2 k_{l}+1\right),
$$

with eigenfunctions corresponding to (3.1). In particular, in the isotropic case when $B_{l}=B>0$ for all $l$, for two multi-indices $k, k^{\prime} \in \mathbb{N}_{0}^{d}$, if $|k|=\left|k^{\prime}\right|$ then $\lambda_{k}=\lambda_{k^{\prime}}$ so that the spectrum of $\mathcal{L}$ consists of eigenvalues of the form $\lambda_{m}=B(2 m+1)$ with $m \in \mathbb{N}_{0}$. We refer e.g. to [47] and references therein for more details on the spectral analysis of this case.

\subsection{Regular Elliptic Boundary Value Problems}

Let $\mathcal{L}$ be a realisation in $L^{2}(\Omega)$ of a regular elliptic boundary value problem, i.e. such that the underlying differential operator is uniformly elliptic and has smooth coefficients on an open bounded set $\Omega \subset \mathbb{R}^{n}$, and that the boundary conditions determining $\mathcal{L}$ are also regular in some sense. Suppose that $\mathcal{L}$ is a positive elliptic operator, so that it has a basis of eigenfunctions in $L^{2}(\Omega)$.

\subsection{Sums of Squares on Compact Lie Groups}

Let $G$ be a compact Lie group and let $X_{1}, \ldots, X_{k}$ be a basis of left-invariant vector fields satisfying the Hörmander sums of squares condition. Let

$$
\mathcal{L}=-\sum_{j=1}^{k} X_{j}^{2}
$$


be the (positive) sub-Laplacian. Then $\mathcal{L}$ has a discrete spectrum which can be related to the spectrum of the bi-invariant Laplacian on $G$, see [24] for the estimates, also involving the representations of $G$.

We refer to [24] for a discussion on the spectral properties and their history in this case. The cases (I.1)-(I.4) have been partially analysed in [24], and the results in cases (II.1)-(II.2) extend them to the case of less regular propagation speeds.

We refer to [51] for questions related to the Fourier analysis on compact Lie groups.

\subsection{Weighted Sub-Laplacians and Sub-Riemannian Ornstein-Uhlenbeck Operators on H-Type and Métivier Groups}

Let $G$ be a Métivier group and let $d$ and $\nabla_{X}$ be a homogeneous norm and the horizontal gradient on $G$, respectively. Let $\mathcal{L}_{\alpha}$ be the weighted sub-Laplacian associated to the Dirichlet form

$$
f \mapsto \int_{G}\left|\nabla_{X} f(x)\right|^{2} e^{-\frac{1}{2} d(x)^{\alpha}} d x .
$$

If $G=\mathbb{R}^{n}$ and $d$ is the Euclidean norm, then for $\alpha=2$, the operator $\mathcal{L}_{2}$ is the classical Ornstein-Uhlenbeck operator. Such operators and their properties have been also intensively studied in sub-Riemannian settings. Thus, in [33] it was shown that if $G$ is an H-type group and $d$ is the Carnot-Carathéodory norm, then the operators $\mathcal{L}_{\alpha}$ have discrete spectrum for $\alpha>1$. If $G$ is a general Métivier group and $d$ is the Kaplan norm, then it was shown in [8] that $\mathcal{L}_{\alpha}$ has discrete spectrum if and only if $\alpha>2$. We refer to e.g. [6] for definitions of these groups and the corresponding norms.

\subsection{Operators on Manifolds with Boundary}

Let $M$ be a manifold with (possibly irregular) boundary $\partial M$. Let $\mathcal{L}_{0}$ be an operator densely defined in $L^{2}(M)$, with discrete spectrum and the eigenfunctions forming a Riesz basis in $L^{2}(M)$.

If $M$ is a closed manifold (i.e. compact without boundary) and $\mathcal{L}_{0}$ is a positive elliptic pseudo-differential operator on $X$ then $\mathcal{L}=\left|\mathcal{L}_{0}\right|=\mathcal{L}_{0}$ in both the operator sense and in the sense explained in the introduction. In this case the basis of the eigenfunctions of $\mathcal{L}$ can be chosen to be orthonormal.

The operator does not have to be elliptic, for example, if we take a family $X_{1}, \ldots, X_{k}$ of smooth vector fields on $M$ satisfying the Hörmander condition, such that the necessarily positive spectrum of the operator

$$
\mathcal{L}=\sum_{j=1}^{k} X_{j} X_{j}^{*}
$$

corresponds to a basis in $L^{2}(M)$, then it satisfies our assumption. In the case of $M$ being a compact Lie group and left-invariant vector fields $X_{j}$, this recaptures the example in Sect. 3.5. 
Another example here may be the operator $\mathcal{L}=i \frac{d}{d x}$ on the manifold $M=[0,1]$ equipped with periodic boundary conditions $f(0)=f(1)$. This can be regarded as a special case of the situation above since in this case $M$ can be identified with the circle.

However, if we take the operator

$$
\mathcal{L}_{0}=i \frac{d}{d x}
$$

on the manifold $M=[0,1]$ equipped with boundary conditions

$$
h f(0)=f(1)
$$

for a fixed $h>0, h \neq 1$, it is no longer self-adjoint. Its eigenvalues are given by

$$
\lambda_{\xi}=-i \ln h+2 \pi \xi, \quad \xi \in \mathbb{Z},
$$

corresponding to eigenfunctions

$$
e_{\xi}(x)=h^{x} e^{2 \pi i x \xi},
$$

which are not orthogonal. We note here that $\mathcal{L}=\left|\mathcal{L}_{0}\right| \neq \mathcal{L}_{0}$ makes sense in the symbolic sense of the introduction but not as a composition of $\mathcal{L}_{0}$ with $\mathcal{L}_{0}^{*}$ which can not be composed having different domains. The function spaces, for example the Sobolev spaces $H_{\mathcal{L}}^{s}$ and $H_{\mathcal{L}_{0}}^{s}$ are comparable since the asymptotic distribution of $\lambda_{\xi}$ and $\left|\lambda_{\xi}\right|$ is the same.

The biorthogonal (nonharmonic) Fourier and symbolic analysis of such operators is still possible and was developed in $[53,55]$ in a general setting, to which we refer for further details.

\subsection{Differential Operators with Strong Regular Boundary Conditions}

We finish the list of examples with another non-self-adjoint operator, following [53]. Let $\mathrm{O}^{(m)}$ be an ordinary differential operator in $L^{2}(0,1)$ of order $m$ generated by the differential expression

$$
l(y) \equiv y^{(m)}(x)+\sum_{k=0}^{m-1} p_{k}(x) y^{(k)}(x), \quad 0<x<1,
$$

with coefficients

$$
p_{k} \in C^{k}[0,1], \quad k=0,1, \ldots, m-1,
$$

and boundary conditions

$$
U_{j}(y) \equiv V_{j}(y)+\sum_{s=0}^{k_{j}} \int_{0}^{1} y^{(s)}(t) \rho_{j s}(t) d t=0, \quad j=1, \ldots, m,
$$


where

$$
V_{j}(y) \equiv \sum_{s=0}^{j}\left[\alpha_{j s} y^{\left(k_{s}\right)}(0)+\beta_{j s} y^{\left(k_{s}\right)}(1)\right],
$$

with $\alpha_{j s}$ and $\beta_{j s}$ some real numbers, and $\rho_{j s} \in L^{2}(0,1)$ for all $j$ and $s$.

Furthermore, we suppose that the boundary conditions (3.8) are normed and strongly regular in the sense considered by Shkalikov in [59]. Then it can be shown that the eigenvalues have the same algebraic and geometric multiplicities and, after a suitable adaption for our case, we have

Theorem 3.1. ([59]) The eigenfunctions of the operator $\mathrm{O}^{(m)}$ with strong regular boundary conditions (3.8) form a Riesz basis in $L^{2}(0,1)$.

In the monograph of Naimark [42] the spectral properties of differential operators generated by the differential expression (3.7) with the boundary conditions (3.8) without integral terms were considered. The statement as in Theorem 3.1 was established in this setting, with the asymptotic formula for the Weyl eigenvalue counting function $N(\lambda)$ in the form

$$
N(\lambda) \sim C \lambda^{1 / m} \text { as } \lambda \rightarrow+\infty .
$$

\section{Main Results, Part II: Very Weak Solutions}

We now describe the notion of very weak solutions and formulate the corresponding results for distributions $a \in \mathcal{D}^{\prime}([0, T])$ and $f \in \mathcal{D}^{\prime}([0, T]) \bar{\otimes} H_{\mathcal{L}}^{-\infty}$. The first main idea is to start from the distributional coefficient $a$ and the source term $f$ to regularise them by convolution with a suitable mollifier $\psi$ obtaining families of smooth functions $\left(a_{\varepsilon}\right)_{\varepsilon}$ and $\left(f_{\varepsilon}\right)_{\varepsilon}$, namely

$$
a_{\varepsilon}=a * \psi_{\omega(\varepsilon)}, \quad f_{\varepsilon}=f(\cdot) * \psi_{\omega(\varepsilon)},
$$

where

$$
\psi_{\omega(\varepsilon)}(t)=\omega(\varepsilon)^{-1} \psi(t / \omega(\varepsilon))
$$

and $\omega(\varepsilon)$ is a positive function converging to 0 as $\varepsilon \rightarrow 0$ to be chosen later. Here $\psi$ is a Friedrichs-mollifier, i.e.

$$
\psi \in C_{0}^{\infty}(\mathbb{R}), \psi \geq 0 \text { and } \int \psi=1
$$

It turns out that the net $\left(a_{\varepsilon}\right)_{\varepsilon}$ is $C^{\infty}$-moderate, in the sense that its $C^{\infty}$-seminorms can be estimated by a negative power of $\varepsilon$. More precisely, we will make use of the following notions of moderateness.

In the sequel, the notation $K \Subset \mathbb{R}$ means that $K$ is a compact set in $\mathbb{R}$. 
Definition 4.1. (i) A net of functions $\left(f_{\varepsilon}\right)_{\varepsilon} \in C^{\infty}(\mathbb{R})^{(0,1]}$ is said to be $C^{\infty}$ moderate if for all $K \Subset \mathbb{R}$ and for all $\alpha \in \mathbb{N}_{0}$ there exist $N \in \mathbb{N}_{0}$ and $c>0$ such that

$$
\sup _{t \in K}\left|\partial^{\alpha} f_{\varepsilon}(t)\right| \leq c \varepsilon^{-N-\alpha},
$$

for all $\varepsilon \in(0,1]$.

(ii) A net of functions $\left(u_{\varepsilon}\right)_{\varepsilon} \in C^{\infty}\left([0, T] ; H_{\mathcal{L}}^{s}\right)^{(0,1]}$ is $C^{\infty}\left([0, T] ; H_{\mathcal{L}}^{s}\right)$-moderate if there exist $N \in \mathbb{N}_{0}$ and $c_{k}>0$ for all $k \in \mathbb{N}_{0}$ such that

$$
\left\|\partial_{t}^{k} u_{\varepsilon}(t, \cdot)\right\|_{H_{\mathcal{L}}^{s}} \leq c_{k} \varepsilon^{-N-k},
$$

for all $t \in[0, T]$ and $\varepsilon \in(0,1]$.

(iii) We say that a net of functions $\left(u_{\varepsilon}\right)_{\varepsilon} \in C^{\infty}\left([0, T] ; H_{(s)}^{-\infty}\right)^{(0,1]}$ is $C^{\infty}([0, T]$; $H_{(s)}^{-\infty}$ )-moderate if there exists $\eta>0$ and, for all $p \in \mathbb{N}_{0}$ there exist $c_{p}>0$ and $N_{p}>0$ such that

$$
\left\|\mathrm{e}^{-\eta \mathcal{L}^{\frac{1}{2 s}}} \partial_{t}^{p} u_{\varepsilon}(t, \cdot)\right\|_{\mathcal{H}} \leq c_{p} \varepsilon^{-N_{p}-p},
$$

for all $t \in[0, T]$ and $\varepsilon \in(0,1]$.

We note that the conditions of moderateness are natural in the sense that regularisations of distributions are moderate, namely we can regard

compactly supported distributions $\mathcal{E}^{\prime}(\mathbb{R}) \subset\left\{C^{\infty}\right.$-moderate families $\}$

by the structure theorems for distributions.

Thus, while a solution to the Cauchy problems may not exist in the space of distributions on the left hand side of (4.2), it may still exist (in a certain appropriate sense) in the space on its right hand side. The moderateness assumption will be crucial allowing to recapture the solution as in (2.3) should it exist. However, we note that regularisation with standard Friedrichs mollifiers will not be sufficient, hence the introduction of a family $\omega(\varepsilon)$ in the above regularisations.

We can now introduce a notion of a 'very weak solution' for the Cauchy problem

$$
\left\{\begin{aligned}
\partial_{t}^{2} u(t)+a(t) \mathcal{L} u(t) & =f(t), t \in[0, T], \\
u(0) & =u_{0} \in \mathcal{H}, \\
\partial_{t} u(0) & =u_{1} \in \mathcal{H} .
\end{aligned}\right.
$$

Definition 4.2. Let $s \in \mathbb{R}$.

(i) The net

$$
\left(u_{\varepsilon}\right)_{\varepsilon} \subset C^{\infty}\left([0, T] ; H_{\mathcal{L}}^{s}\right)
$$

is a very weak solution of $H^{s}$-type of the Cauchy problem (4.3) if there exist $C^{\infty}$-moderate regularisation $a_{\varepsilon}$ of the coefficient $a$, $C^{\infty}\left([0, T] ; H_{\mathcal{L}}^{s}\right)$-moderate regularisation $f_{\varepsilon}(t)$ of the source term $f(t)$, 
such that $\left(u_{\varepsilon}\right)_{\varepsilon}$ solves the regularised problem

$$
\left\{\begin{aligned}
\partial_{t}^{2} u_{\varepsilon}(t)+a_{\varepsilon}(t) \mathcal{L} u_{\varepsilon}(t) & =f_{\varepsilon}(t), t \in[0, T], \\
u_{\varepsilon}(0) & =u_{0} \in \mathcal{H}, \\
\partial_{t} u_{\varepsilon}(0) & =u_{1} \in \mathcal{H},
\end{aligned}\right.
$$

for all $\varepsilon \in(0,1]$, and is $C^{\infty}\left([0, T] ; H_{\mathcal{L}}^{s}\right)$-moderate.

(ii) We say that the net

$$
\left(u_{\varepsilon}\right)_{\varepsilon} \subset C^{\infty}\left([0, T] ; H_{(s)}^{-\infty}\right)
$$

is a very weak solution of $H_{(s)}^{-\infty}$-type of the Cauchy problem (4.3) if there exist $C^{\infty}$-moderate regularisation $a_{\varepsilon}$ of the coefficient $a$,

$C^{\infty}\left([0, T] ; H_{(s)}^{-\infty}\right)$-moderate regularisation $f_{\varepsilon}(t)$ of the source term $f(t)$,

such that $\left(u_{\varepsilon}\right)_{\varepsilon}$ solves the regularised problem (4.4) for all $\varepsilon \in(0,1]$, and is $C^{\infty}\left([0, T] ; H_{(s)}^{-\infty}\right)$-moderate.

We note that according to Theorem 2.1 the regularised Cauchy problem (4.4) has a unique solution satisfying estimate (2.3).

In [25] Claudia Garetto and the first-named author studied weakly hyperbolic second order equations with time-dependent irregular coefficients, assuming that the coefficients are distributions. For such equations, the authors of [25] introduced the notion of a 'very weak solution' adapted to the type of solutions that exist for regular coefficients. We now apply a modification of this notion to the Cauchy problem (4.3). In fact, in our particular setting, the condition that the distribution $a$ is nonnegative implies that it has to be a Radon measure. However, we will not be making much use of this observation, especially since we could not make the same conclusion on the behaviour of the source term $f$ with respect to $t$.

In the case of the Landau Hamiltonian with irregular (distributional) electromagnetic fields the Sobolev type very weak solutions have been constructed in [54] where we proved the first part of the following result in that case.

In the following theorem we assume that $a$ is a nonnegative or a strictly positive distribution. The strict positivity means that there exists a constant $a_{0}>0$ such that $a-a_{0}$ is a positive distribution. In other words,

$$
a \geq a_{0}>0,
$$

where $a \geq a_{0}$ means that $a-a_{0} \geq 0$, i.e. $\left\langle a-a_{0}, \psi\right\rangle \geq 0$ for all $\psi \in C_{0}^{\infty}(\mathbb{R})$, $\psi \geq 0$.

The main results of this part of the paper can be summarised as the following solvability statement complemented by the uniqueness and consistency in Theorems 7.2 and 4.4:

Theorem 4.3. (Existence) Let $s \in \mathbb{R}$.

(II.1) Let the coefficient a of the Cauchy problem (4.3) be a positive distribution with compact support included in $[0, T]$, such that $a \geq a_{0}$ for some constant $a_{0}>0$. Let the Cauchy data $u_{0}, u_{1}$ be in $H_{\mathcal{L}}^{s}$ and the source term $f \in$ $\mathcal{D}^{\prime}([0, T]) \bar{\otimes} H_{\mathcal{L}}^{s}$. Then the Cauchy problem (4.3) has a very weak solution of $H^{s}$-type. 
(II.2) Let the coefficient a of the Cauchy problem (4.3) be a nonnegative distribution with compact support included in $[0, T]$, such that $a \geq 0$. Let the Cauchy data $u_{0}, u_{1}$ be in $H_{(s)}^{-\infty}$ and the source term $f \in \mathcal{D}^{\prime}([0, T]) \bar{\otimes} H_{(s)}^{-\infty}$. Then the Cauchy problem (4.3) has a very weak solution of $H_{(s)}^{-\infty}$-type.

In Theorem 7.2 we show that the very weak solution is unique in an appropriate sense, but now let us formulate the theorem saying that very weak solutions recapture the classical solutions in the case the latter exist. For example, this happens under conditions of Theorems 2.1 and 2.3 (b). So, we can compare the solution given by Theorems 2.1 and 2.3 (b) with the very weak solutions in Theorem 4.3 under assumptions when Theorems 2.1 and 2.3 (b) hold.

As usual, by $L_{1}^{\infty}([0, T])$ we denote the space of bounded functions on $[0, T]$ with the derivative also in $L^{\infty}$.

Theorem 4.4. (Consistency-1)

(II.1) Assume that $a \in L_{1}^{\infty}([0, T])$ is such that $a(t) \geq a_{0}>0$. Let $s \in \mathbb{R}$ and consider the Cauchy problem

$$
\left\{\begin{aligned}
\partial_{t}^{2} u(t)+a(t) \mathcal{L} u(t) & =f(t), t \in[0, T], \\
u(0) & =u_{0} \in \mathcal{H}, \\
\partial_{t} u(0) & =u_{1} \in \mathcal{H},
\end{aligned}\right.
$$

with $\left(u_{0}, u_{1}\right) \in H_{\mathcal{L}}^{1+s} \times H_{\mathcal{L}}^{s}$ and the source term $f \in C\left([0, T] ; H_{\mathcal{L}}^{s}\right)$. Let $u$ be a very weak solution of $H^{s}$-type of (4.5). Then for any regularising families $a_{\varepsilon}$ and $f_{\varepsilon}$ in Definition 4.2 , any representative $\left(u_{\varepsilon}\right)_{\varepsilon}$ of $u$ converges in $C\left([0, T] ; H_{\mathcal{L}}^{1+s}\right) \cap C^{1}\left([0, T] ; H_{\mathcal{L}}^{s}\right)$ as $\varepsilon \rightarrow 0$ to the unique classical solution in $C\left([0, T] ; H_{\mathcal{L}}^{1+s}\right) \cap C^{1}\left([0, T] ; H_{\mathcal{L}}^{s}\right)$ of the Cauchy problem (4.5) given by Theorem 2.1 .

(II.2) Assume that $a \in C^{\ell}([0, T])$ with $\ell \geq 2$ is such that $a(t) \geq 0$. Let

$$
1 \leq s<1+\frac{\ell}{2}
$$

and consider the Cauchy problem (4.5) with $u_{0}, u_{1} \in H_{(s)}^{-\infty}$ as well as $f \in C\left([0, T] ; H_{(s)}^{-\infty}\right)$. Let u be a very weak solution of $H_{(s)}^{-\infty}$-type of (4.5). Then for any regularising families $a_{\varepsilon}$ and $f_{\varepsilon}$ in Definition 4.2, any representative $\left(u_{\varepsilon}\right)_{\varepsilon}$ of u converges in $C^{2}\left([0, T] ; H_{(s)}^{-\infty}\right)$ as $\varepsilon \rightarrow 0$ to the unique classical solution in $C^{2}\left([0, T] ; H_{(s)}^{-\infty}\right)$ of the Cauchy problem (4.5) given by Theorem $2.3(b)$.

In a similar way, we can prove additional consistency 'cases' of Theorem 4.4, corresponding to Theorem 2.2 (b) and Theorem 2.4 (b):

Theorem 4.5. (Consistency-2)

(II.3) Assume that $a(t) \geq a_{0}>0$ and that $a \in C^{\alpha}([0, T])$ with $0<\alpha<1$. Let

$$
1 \leq s<1+\frac{\alpha}{1-\alpha}
$$


and consider the Cauchy problem

$$
\left\{\begin{aligned}
\partial_{t}^{2} u(t)+a(t) \mathcal{L} u(t) & =f(t), t \in[0, T], \\
u(0) & =u_{0} \in \mathcal{H}, \\
\partial_{t} u(0) & =u_{1} \in \mathcal{H},
\end{aligned}\right.
$$

with $u_{0}, u_{1} \in H_{(s)}^{-\infty}$ and $f \in C\left([0, T] ; H_{(s)}^{-\infty}\right)$. Let $u$ be a very weak solution of $H_{(s)}^{-\infty}$-type of (4.6). Then for any regularising families $a_{\varepsilon}$ and $f_{\varepsilon}$ in Definition 4.2, any representative $\left(u_{\varepsilon}\right)_{\varepsilon}$ of u converges in $C^{2}\left([0, T] ; H_{(s)}^{-\infty}\right)$ as $\varepsilon \rightarrow 0$ to the unique classical solution in $C^{2}\left([0, T] ; H_{(s)}^{-\infty}\right)$ of the Cauchy problem (4.6) given by Theorem $2.2(b)$.

(II.4) Assume that $a(t) \geq 0$ and that $a \in C^{\alpha}([0, T])$ with $0<\alpha<2$. Let

$$
1 \leq s<1+\frac{\alpha}{2}
$$

and consider the Cauchy problem (4.6) with $u_{0}, u_{1} \in H_{(s)}^{-\infty}$ as well as $f \in C\left([0, T] ; H_{(s)}^{-\infty}\right)$. Let $u$ be a very weak solution of $H_{(s)}^{-\infty}$-type of (4.6). Then for any regularising families $a_{\varepsilon}$ and $f_{\varepsilon}$ in Definition 4.2, any representative $\left(u_{\varepsilon}\right)_{\varepsilon}$ of $u$ converges in $C^{2}\left([0, T] ; H_{(s)}^{-\infty}\right)$ as $\varepsilon \rightarrow 0$ to the unique classical solution in $C^{2}\left([0, T] ; H_{(s)}^{-\infty}\right)$ of the Cauchy problem (4.6) given by Theorem 2.4 (b).

The proofs of these results will be given in Sect. 7. The uniqueness of the very weak solutions will be formulated in Theorem 7.2.

\section{5. $\mathcal{L}$-Fourier Analysis}

In this section we recall the necessary elements of the global Fourier analysis that has been developed in [53] (also see [55], and its applications to the spectral properties of operators in [19]). The space

$$
H_{\mathcal{L}}^{\infty}:=\operatorname{Dom}\left(\mathcal{L}^{\infty}\right)
$$

is called the space of test functions for $\mathcal{L}$. Here we define

$$
\operatorname{Dom}\left(\mathcal{L}^{\infty}\right):=\bigcap_{k=1}^{\infty} \operatorname{Dom}\left(\mathcal{L}^{k}\right),
$$

where $\operatorname{Dom}\left(\mathcal{L}^{k}\right)$ is the domain of the operator $\mathcal{L}^{k}$, in turn defined as

$$
\operatorname{Dom}\left(\mathcal{L}^{k}\right):=\left\{f \in \mathcal{H}: \mathcal{L}^{j} f \in \operatorname{Dom}(\mathcal{L}), \quad j=0,1,2, \ldots, k-1\right\} .
$$

The Fréchet topology of $H_{\mathcal{L}}^{\infty}$ is given by the family of semi-norms

$$
\|\varphi\|_{H_{\mathcal{L}}^{k}}:=\max _{j \leq k}\left\|\mathcal{L}^{j} \varphi\right\|_{\mathcal{H}}, \quad k \in \mathbb{N}_{0}, \varphi \in H_{\mathcal{L}}^{\infty}
$$


Analogously to the operator $\mathcal{L}^{*}(\mathcal{H}$-conjugate to $\mathcal{L})$, we introduce the space

$$
H_{\mathcal{L}^{*}}^{\infty}:=\operatorname{Dom}\left(\left(\mathcal{L}^{*}\right)^{\infty}\right)
$$

of test functions for $\mathcal{L}^{*}$, and we define

$$
\operatorname{Dom}\left(\left(\mathcal{L}^{*}\right)^{\infty}\right):=\bigcap_{k=1}^{\infty} \operatorname{Dom}\left(\left(\mathcal{L}^{*}\right)^{k}\right),
$$

where $\operatorname{Dom}\left(\left(\mathcal{L}^{*}\right)^{k}\right)$ is the domain of the operator $\left(\mathcal{L}^{*}\right)^{k}$, in turn defined as

$$
\operatorname{Dom}\left(\left(\mathcal{L}^{*}\right)^{k}\right):=\left\{f \in \mathcal{H}:\left(\mathcal{L}^{*}\right)^{j} f \in \operatorname{Dom}\left(\mathcal{L}^{*}\right), \quad j=0,1,2, \ldots, k-1\right\} .
$$

The Fréchet topology of $C_{\mathcal{L}^{*}}^{\infty}$ is given by the family of semi-norms

$$
\|\varphi\|_{H_{\mathcal{L}^{*}}^{k}}:=\max _{j \leq k}\left\|\left(\mathcal{L}^{*}\right)^{j} \varphi\right\|_{\mathcal{H}}, \quad k \in \mathbb{N}_{0}, \varphi \in C_{\mathcal{L}^{*}}^{\infty} .
$$

The space

$$
H_{\mathcal{L}}^{-\infty}:=\mathcal{L}\left(H_{\mathcal{L}^{*}}^{\infty}, \mathbb{C}\right)
$$

of linear continuous functionals on $H_{\mathcal{L}^{*}}^{\infty}$ is called the space of $\mathcal{L}$-distributions. We can understand the continuity here in terms of the topology (5.2). For $w \in H_{\mathcal{L}}^{-\infty}$ and $\varphi \in H_{\mathcal{L}^{*}}^{\infty}$, we shall write

$$
w(\varphi)=\langle w, \varphi\rangle
$$

For any $\psi \in H_{\mathcal{L}}^{\infty}$, the functional

$$
H_{\mathcal{L}^{*}}^{\infty} \ni \varphi \mapsto(\psi, \varphi)
$$

is an $\mathcal{L}$-distribution, which gives an embedding $\psi \in H_{\mathcal{L}}^{\infty} \hookrightarrow H_{\mathcal{L}}^{-\infty}$.

Now the space

$$
H_{\mathcal{L}^{*}}^{-\infty}:=\mathcal{L}\left(H_{\mathcal{L}}^{\infty}, \mathbb{C}\right)
$$

of linear continuous functionals on $H_{\mathcal{L}}^{\infty}$ is called the space of $\mathcal{L}^{*}$-distributions. We can understand the continuity here in terms of the topology (5.1). For $w \in H_{\mathcal{L}^{*}}^{-\infty}$ and $\varphi \in H_{\mathcal{L}}^{\infty}$, we shall also write

$$
w(\varphi)=\langle w, \varphi\rangle .
$$

For any $\psi \in H_{\mathcal{L}^{*}}^{\infty}$, the functional

$$
H_{\mathcal{L}}^{\infty} \ni \varphi \mapsto(\psi, \varphi)
$$

is an $\mathcal{L}^{*}$-distribution, which gives an embedding $\psi \in H_{\mathcal{L}^{*}}^{\infty} \hookrightarrow H_{\mathcal{L}^{*}}^{-\infty}$.

Since the system of eigenfunctions $\left\{e_{\xi}: \xi \in \mathcal{I}\right\}$ of the operator $\mathcal{L}$ is a Riesz basis in $\mathcal{H}$ then its biorthogonal system $\left\{e_{\xi}^{*}: \xi \in \mathcal{I}\right\}$ is also a Riesz basis in $\mathcal{H}$ (see e.g. Bari [4], as well as Gelfand [26]). Note that the function $e_{\xi}^{*}$ is an eigenfunction 
of the operator $\mathcal{L}^{*}$ corresponding to the eigenvalue $\bar{\lambda}_{\xi}$ for each $\xi \in \mathcal{I}$. They satisfy the orthogonality relations

$$
\left(e_{\xi}, e_{\eta}^{*}\right)=\delta \xi \eta,
$$

where $\delta_{\xi \eta}$ is the Kronecker delta.

Let $\mathcal{S}(\mathcal{I})$ denote the space of rapidly decaying functions $\varphi: \mathcal{I} \rightarrow \mathbb{C}$. That is, $\varphi \in \mathcal{S}(\mathcal{I})$ if for any $m<\infty$ there exists a constant $C_{\varphi, m}$ such that

$$
|\varphi(\xi)| \leq C_{\varphi, m}\langle\xi\rangle^{-m}
$$

holds for all $\xi \in \mathcal{I}$, where we denote

$$
\langle\xi\rangle:=\left(1+\left|\lambda_{\xi}\right|\right)^{1 / 2} .
$$

The topology on $\mathcal{S}(\mathcal{I})$ is given by the seminorms $p_{k}$, where $k \in \mathbb{N}_{0}$ and

$$
p_{k}(\varphi):=\sup _{\xi \in \mathcal{I}}\langle\xi\rangle^{k}|\varphi(\xi)| .
$$

We now define the $\mathcal{L}$-Fourier transform on $H_{\mathcal{L}}^{\infty}$ as the mapping

$$
\left(\mathcal{F}_{\mathcal{L}} f\right)(\xi)=(f \mapsto \widehat{f}): H_{\mathcal{L}}^{\infty} \rightarrow \mathcal{S}(\mathcal{I})
$$

by the formula

$$
\widehat{f}(\xi):=\left(\mathcal{F}_{\mathcal{L}} f\right)(\xi)=\left(f, e_{\xi}^{*}\right),
$$

and define the $\mathcal{L}^{*}$-Fourier transform on $H_{\mathcal{L}^{*}}^{\infty}$ as the mapping

$$
\left(\mathcal{F}_{\mathcal{L}^{*}} g\right)(\xi)=\left(g \mapsto \widehat{g}_{*}\right): H_{\mathcal{L}^{*}}^{\infty} \rightarrow \mathcal{S}(\mathcal{I})
$$

by the formula

$$
\widehat{g}_{*}(\xi):=\left(\mathcal{F}_{\mathcal{L}^{*}} g\right)(\xi)=\left(g, e_{\xi}\right) .
$$

The $\mathcal{L}$-Fourier transform $\mathcal{F}_{\mathcal{L}}$ is a bijective homeomorphism from $H_{\mathcal{L}}^{\infty}$ to $\mathcal{S}(\mathcal{I})$. Its inverse

$$
\mathcal{F}_{\mathcal{L}}^{-1}: \mathcal{S}(\mathcal{I}) \rightarrow H_{\mathcal{L}}^{\infty}
$$

is given by

$$
\left(\mathcal{F}_{\mathcal{L}}^{-1} h\right)=\sum_{\xi \in \mathcal{I}} h(\xi) e_{\xi}, \quad h \in \mathcal{S}(\mathcal{I}),
$$

so that the Fourier inversion formula becomes

$$
f=\sum_{\xi \in \mathcal{I}} \widehat{f}(\xi) e_{\xi} \quad \text { for all } f \in H_{\mathcal{L}}^{\infty} .
$$

Also the $\mathcal{L}^{*}$-Fourier transform $\mathcal{F}_{\mathcal{L}^{*}}$ is a bijective homeomorphism from $H_{\mathcal{L}^{*}}^{\infty}$ to $\mathcal{S}(\mathcal{I})$. Its inverse

$$
\mathcal{F}_{\mathcal{L}^{*}}^{-1}: \mathcal{S}(\mathcal{I}) \rightarrow H_{\mathcal{L}^{*}}^{\infty}
$$


is given by

$$
\left(\mathcal{F}_{\mathcal{L}^{*}}^{-1} g\right)=\sum_{\xi \in \mathcal{I}} g(\xi) e_{\xi}^{*}, \quad g \in \mathcal{S}(\mathcal{I}),
$$

so that the $\mathcal{L}^{*}$-Fourier inversion formula becomes

$$
h=\sum_{\xi \in \mathcal{I}} \widehat{h}_{*}(\xi) e_{\xi}^{*} \quad \text { for all } \quad h \in H_{\mathcal{L} *}^{\infty} .
$$

The Plancherel's identity takes the form

$$
\|f\|_{\mathcal{H}}=\left(\sum_{\xi \in \mathcal{I}} \widehat{f}(\xi) \widehat{\widehat{f}_{*}(\xi)}\right)^{1 / 2} .
$$

We note that since systems $\left\{e_{\xi}\right\}$ and $\left\{e_{\xi}^{*}\right\}$ are Riesz bases, we can also compare $\mathcal{H}$-norms of functions with sums of squares of Fourier coefficients. The following statement follows from the work of Bari [4, Theorem 9]:

Lemma 5.1. There exist constants $k, K, m, M>0$ such that for every $f \in \mathcal{H}$ we have

$$
m^{2}\|f\|_{\mathcal{H}}^{2} \leq \sum_{\xi \in \mathcal{I}}|\widehat{f}(\xi)|^{2} \leq M^{2}\|f\|_{\mathcal{H}}^{2}
$$

and

$$
k^{2}\|f\|_{\mathcal{H}}^{2} \leq \sum_{\xi \in \mathcal{I}}\left|\widehat{f_{*}}(\xi)\right|^{2} \leq K^{2}\|f\|_{\mathcal{H}}^{2} .
$$

Hence, Lemma 5.1 shows that

$$
\|f\|_{1, \mathcal{H}}:=\sqrt{(f, f)}=\left(\sum_{\xi \in \mathcal{I}} \widehat{f}(\xi) \widehat{\widehat{f}_{*}(\xi)}\right)^{1 / 2}
$$

and

$$
\|f\|_{2, \mathcal{H}}:=\left(\sum_{\xi \in \mathcal{I}}|\widehat{f}(\xi)|^{2}\right)^{1 / 2}
$$

and

$$
\|f\|_{3, \mathcal{H}}:=\left(\sum_{\xi \in \mathcal{I}}\left|\widehat{f}_{*}(\xi)\right|^{2}\right)^{1 / 2}
$$

are equivalent norms. Indeed, we could use any of them.

Now we are going to introduce Sobolev spaces induced by the operator $\mathcal{L}$. For this aim we will use $\|\cdot\|_{2, \mathcal{H}}$-norm and, briefly, write $\|\cdot\|_{\mathcal{H}}$. In fact, it does not matter what norm we use because, as a result, we get equivalent Sobolev norms. 
In general, given a linear continuous operator $L: H_{\mathcal{L}}^{\infty} \rightarrow H_{\mathcal{L}}^{\infty}$ (or even $L$ : $H_{\mathcal{L}}^{\infty} \rightarrow H_{\mathcal{L}}^{-\infty}$ ), under the condition that $e_{\xi}$ does not have zeros, we can define its symbol by

$$
\sigma_{L}(\xi):=e_{\xi}^{-1}\left(L e_{\xi}\right)
$$

In this case it holds that

$$
L f=\sum_{\xi \in \mathcal{I}} \sigma_{L}(\xi) \widehat{f}(\xi) e_{\xi}
$$

The correspondence between operators and symbols is one-to-one. The quantization (5.10) has been extensively studied in $[51,52]$ in the setting of compact Lie groups, and in [53] in the setting of (non-self-adjoint) boundary value problems, to which we may refer for its properties and for the corresponding symbolic calculus. The condition that $e_{\xi}$ do not have zeros can be removed in some sense, see [55]. However, in this paper we do not need such technicalities since we already know the symbols of all the appearing operators.

Consequently, we can also define Sobolev spaces $H_{\mathcal{L}}^{s}$ associated to $\mathcal{L}$. Thus, for any $s \in \mathbb{R}$, we set

$$
H_{\mathcal{L}}^{s}:=\left\{f \in H_{\mathcal{L}}^{-\infty}: \mathcal{L}^{s / 2} f \in \mathcal{H}\right\}
$$

with the norm $\|f\|_{H_{\mathcal{C}}^{s}}:=\left\|\mathcal{L}^{s / 2} f\right\|_{\mathcal{H}}$, which, using Lemma 5.1, we may also understand as

$$
\|f\|_{H_{\mathcal{L}}^{s}}:=\left\|\mathcal{L}^{s / 2} f\right\|_{\mathcal{H}}:=\left(\sum_{\xi \in \mathcal{I}}\left|\sigma_{\mathcal{L}}(\xi)\right|^{s}|\widehat{f}(\xi)|^{2}\right)^{1 / 2},
$$

justifying the expression (2.2) since $\sigma_{\mathcal{L}}(\xi)=\lambda \xi$.

The (Roumieu) Gevrey space $\gamma_{\mathcal{L}}^{s}$ mentioned in (2.4) is defined by the formula

$$
\begin{aligned}
& f \in \gamma_{\mathcal{L}}^{s} \Longleftrightarrow \exists A>0: \\
& \left\|\mathrm{e}^{A \mathcal{L} \frac{1}{2 s}} f\right\|_{\mathcal{H}}^{2}:=\sum_{\xi \in \mathcal{I}}\left|\mathrm{e}^{A\left|\sigma_{\mathcal{L}}(\xi)\right| \frac{1}{2 s}} \widehat{f}(\xi)\right|^{2}=\sum_{\xi \in \mathcal{I}} \mathrm{e}^{2 A\left|\sigma_{\mathcal{L}}(\xi)\right| \frac{1}{2 s}}|\widehat{f}(\xi)|^{2}<\infty .
\end{aligned}
$$

Also, define the Beurling Gevrey space $\gamma_{\mathcal{L}}^{(s)}$ by the formula

$$
\begin{aligned}
& g \in \gamma_{\mathcal{L}}^{(s)} \Longleftrightarrow \forall B>0: \\
& \left\|\mathrm{e}^{B \mathcal{L} \frac{1}{2 s}} g\right\|_{\mathcal{H}}^{2}:=\sum_{\xi \in \mathcal{I}}\left|\mathrm{e}^{B\left|\sigma_{\mathcal{L}}(\xi)\right|^{\frac{1}{2 s}}} \widehat{g}(\xi)\right|^{2}=\sum_{\xi \in \mathcal{I}} \mathrm{e}^{2 B\left|\sigma_{\mathcal{L}}(\xi)\right|^{\frac{1}{2 s}}}|\widehat{g}(\xi)|^{2}<\infty .
\end{aligned}
$$

In the case when $\mathcal{L}$ is the Laplacian (or, more generally, a positive elliptic pseudo-differential operator) on a closed manifold it was shown in [18] that these spaces coincide with the usual Gevrey spaces defined in local coordinates. 
We denote by $H_{s}^{-\infty}$ and $H_{(s)}^{-\infty}$ the spaces of linear continuous functionals on $\gamma_{\mathcal{L}}^{s}$ and $\gamma_{\mathcal{L}}^{(s)}$, respectively. We call them the Gevrey Roumieu ultradistributions and the Gevrey Beurling ultradistributions, respectively. Then, Proposition 13 in [22] can be easily adapted to our case. Hence, the following Fourier characterisations of duals of $\gamma_{\mathcal{L}}^{s}$ and $\gamma_{\mathcal{L}}^{(s)}$ are valid:

Corollary 5.2. We have $u \in H_{s}^{-\infty}$ if and only iffor any $\delta>0$ there exists $C_{\delta}$ such that

$$
|\widehat{u}(\xi)| \leq C_{\delta} \mathrm{e}^{\delta\left|\sigma_{\mathcal{L}}(\xi)\right|^{\frac{1}{2 s}}}
$$

for all $\xi \in \mathcal{I}$. Similarly, a functional $u$ belongs to $H_{(s)}^{-\infty}$ if and only if there are constants $\eta>0$ and $C>0$ such that

$$
|\widehat{u}(\xi)| \leq C \mathrm{e}^{\eta\left|\sigma_{\mathcal{L}}(\xi)\right|^{\frac{1}{2 s}}}
$$

for all $\xi \in \mathcal{I}$.

Again, let us use the Plancherel identity. The Gevrey Roumieu ultradistributions $H_{s}^{-\infty}$ and the Gevrey Beurling ultradistributions $H_{(s)}^{-\infty}$ can be characterized by

$$
\begin{aligned}
& u \in H_{s}^{-\infty} \Longleftrightarrow \forall \delta>0: \\
& \left\|\mathrm{e}^{-\delta \mathcal{L} \frac{1}{2 s}} u\right\|_{\mathcal{H}}^{2}:=\sum_{\xi \in \mathcal{I}}\left|\mathrm{e}^{-\delta \mid \sigma_{\mathcal{L}}(\xi) \frac{1}{2 s}} \widehat{u}(\xi)\right|^{2}=\sum_{\xi \in \mathcal{I}} \mathrm{e}^{-2 \delta\left|\sigma_{\mathcal{L}}(\xi)\right| \frac{1}{2 s}}|\widehat{u}(\xi)|^{2}<\infty,
\end{aligned}
$$

and

$$
\begin{aligned}
& u \in H_{(s)}^{-\infty} \Longleftrightarrow \exists \eta>0: \\
& \left\|\mathrm{e}^{-\eta \mathcal{L}^{\frac{1}{2 s}}} u\right\|_{\mathcal{H}}^{2}:=\sum_{\xi \in \mathcal{I}}\left|\mathrm{e}^{-\eta\left|\sigma_{\mathcal{L}}(\xi)\right|^{\frac{1}{2 s}}} \widehat{u}(\xi)\right|^{2}=\sum_{\xi \in \mathcal{I}} \mathrm{e}^{-2 \eta\left|\sigma_{\mathcal{L}}(\xi)\right|^{\frac{1}{2 s}}}|\widehat{u}(\xi)|^{2}<\infty,
\end{aligned}
$$

respectively.

Let us now briefly discuss the Gevrey space $\gamma_{\mathcal{L}}^{s}$ defined in (5.13). Let us assume that the eigenvalues $\lambda \xi \geq 0$ of the operator $\mathcal{L}$ satisfy the growth condition:

$$
\text { for all } B>0 \text { we have } \sum_{\xi \in \mathcal{I}} \mathrm{e}^{-B \lambda_{\xi}^{\frac{1}{2 s}}}<\infty \text {. }
$$

Then it follows that the space $\gamma_{\mathcal{L}}^{s}$ can be characterised as

$$
f \in \gamma_{\mathcal{L}}^{s} \Longleftrightarrow \exists A>0, C>0: \forall \xi \in \mathcal{I} \quad|\widehat{f}(\xi)| \leq C \mathrm{e}^{-A\left|\sigma_{\mathcal{L}}(\xi)\right| \frac{1}{2 s}}<\infty .
$$

Consequently, we have the following alternative characterisation of the space $\gamma_{\mathcal{L}}^{s}$. 
Proposition 5.3. Assume the condition (5.16). Then we have $f \in \gamma_{\mathcal{L}}^{s}$ if and only if there exist $A, C>0$ such that for every integer $k \in \mathbb{N}_{0}$ we have

$$
\left\|\mathcal{L}^{k} f\right\|_{\mathcal{H}} \leq C A^{2 k}((2 k) !)^{s} .
$$

The "if" part can be proved by an argument similar to that of [17, Theorem $2.3(\mathrm{R})$, "only if" part] or [18, Theorem 2.4]. For the "only if" part see these papers, as well as [20].

A similar description can be given to the space $\gamma_{\mathcal{L}}^{(s)}$ from (5.13).

\section{Proofs of Part I: Theorems 2.1-2.4}

The operator $\mathcal{L}$ has the symbol

$$
\sigma_{\mathcal{L}}(\xi)=\lambda \xi,
$$

taking the $\mathcal{L}$-Fourier transform of (1.1), we obtain the collection of Cauchy problems for $\mathcal{L}$-Fourier coefficients:

$$
\partial_{t}^{2} \widehat{u}(t, \xi)+a(t) \sigma_{\mathcal{L}}(\xi) \widehat{u}(t, \xi)=\widehat{f}(t, \xi), \xi \in \mathcal{I} .
$$

The main point of our further analysis is that we can make an individual treatment of the equations in (6.2). Thus, let us fix $\xi \in \mathcal{I}$, we then study the Cauchy problem

$$
\partial_{t}^{2} \widehat{u}(t, \xi)+a(t) \sigma_{\mathcal{L}}(\xi) \widehat{u}(t, \xi)=\widehat{f}(t, \xi), \quad \widehat{u}(0, \xi)=\widehat{u}_{0}(\xi), \partial_{t} \widehat{u}(0, \xi)=\widehat{u}_{1}(\xi),
$$

with $\xi$ being a parameter, and want to derive estimates for $\widehat{u}(t, \xi)$. Combined with the characterisation (2.2) of Sobolev spaces this will yield the well-posedness results for the original Cauchy problem (1.1).

By setting

$$
v^{2}(\xi):=\left|\sigma_{\mathcal{L}}(\xi)\right|=\lambda \xi,
$$

the equation in (6.3) can be written as

$$
\partial_{t}^{2} \widehat{u}(t, \xi)+a(t) v^{2}(\xi) \widehat{u}(t, \xi)=\widehat{f}(t, \xi) .
$$

We now proceed with a standard reduction to a first order system of this equation and define the corresponding energy. The energy estimates will be given in terms of $t$ and $\nu(\xi)$.

We can now do the natural energy construction for (6.5). We use the transformation

$$
\begin{aligned}
& V_{1}:=i \nu(\xi) \widehat{u}, \\
& V_{2}:=\partial_{t} \widehat{u} .
\end{aligned}
$$

It follows that the equation (6.5) can be written as the first order system

$$
\partial_{t} V(t, \xi)=i \nu(\xi) A(t) V(t, \xi)+F(t, \xi),
$$


where $V$ is the column vector with entries $V_{1}$ and $V_{2}$,

$$
F(t, \xi)=\left(\begin{array}{c}
0 \\
\widehat{f}(t, \xi)
\end{array}\right),
$$

and

$$
A(t)=\left(\begin{array}{cc}
0 & 1 \\
a(t) & 0
\end{array}\right)
$$

The initial conditions $\widehat{u}(0, \xi)=\widehat{u}_{0}(\xi), \partial_{t} \widehat{u}(0, \xi)=\widehat{u}_{1}(\xi)$ are transformed into

$$
V(0, \xi)=\left(\begin{array}{c}
i v(\xi) \widehat{u}_{0}(\xi) \\
\widehat{u}_{1}(\xi)
\end{array}\right)
$$

Note that the matrix $A$ has eigenvalues $\pm \sqrt{a(t)}$ and symmetriser

$$
S(t)=\left(\begin{array}{cc}
a(t) & 0 \\
0 & 1
\end{array}\right) .
$$

By definition of the symmetriser we have that

$$
S A-A^{*} S=0 .
$$

It is immediate to see that

$$
\min _{t \in[0, T]}(a(t), 1)|V|^{2} \leq(S V, V) \leq \max _{t \in[0, T]}(a(t), 1)|V|^{2},
$$

where $(\cdot, \cdot)$ and $|\cdot|$ denote the inner product and the norm in $\mathbb{C}$, respectively.

\subsection{Case I.1: Proof of Theorem 2.1}

Since $a(t)>0, a \in C^{1}([0, T])$, it is clear that there exist constants $a_{0}>0$ and $a_{1}>0$ such that

$$
a_{0}=\min _{t \in[0, T]} a(t) \text { and } a_{1}=\max _{t \in[0, T]} a(t) .
$$

Hence (6.7) implies,

$$
c_{0}|V|^{2}=\min \left(a_{0}, 1\right)|V|^{2} \leq(S V, V) \leq \max \left(a_{1}, 1\right)|V|^{2}=c_{1}|V|^{2},
$$

with $c_{0}, c_{1}>0$. We then define the energy

$$
E(t, \xi):=(S(t) V(t, \xi), V(t, \xi))
$$

and

$$
\begin{aligned}
\partial_{t} E(t, \xi) & =\left(\partial_{t} S V, V\right)+\left(S \partial_{t} V, V\right)+\left(S V, \partial_{t} V\right) \\
& =\left(\partial_{t} S V, V\right)+i v(\xi)(S A V, V)+(S F, V)-i v(\xi)(S V, A V)+(S V, F) \\
& =\left(\partial_{t} S V, V\right)+i v(\xi)\left(\left(S A-A^{*} S\right) V, V\right)+2 \operatorname{Re}(S F, V) \\
& =\left(\partial_{t} S V, V\right)+2 \operatorname{Re}(S F, V)
\end{aligned}
$$




$$
\begin{aligned}
& \leq\left(\left\|\partial_{t} S\right\|+1\right)|V|^{2}+\|S F\|^{2} \\
& \leq \max \left(\left\|\partial_{t} S\right\|+1,\|S\|^{2}\right)\left(|V|^{2}+|F|^{2}\right) \\
& \leq C_{1} E(t, \xi)+C_{2}|F|^{2},
\end{aligned}
$$

with some constants $C_{1}$ and $C_{2}$. An application of Gronwall's lemma combined with the estimates (6.8) implies

$$
|V|^{2} \leq c_{0}^{-1} E(t, \xi) \leq C_{1}\left|V_{0}\right|^{2}+C_{2} \sup _{0 \leq t \leq T}|F(t, \xi)|^{2},
$$

which is valid for all $t \in[0, T]$ and $\xi$ with 'new' constants $C_{1}$ and $C_{2}$ depending on $T$. Hence

$$
v^{2}(\xi)|\widehat{u}(t, \xi)|^{2}+\left|\partial_{t} \widehat{u}(t, \xi)\right|^{2} \leq C_{1}^{\prime}\left(v^{2}(\xi)\left|\widehat{u}_{0}(\xi)\right|^{2}+\left|\widehat{u}_{1}(\xi)\right|^{2}+\sup _{0 \leq t \leq T}|\widehat{f}(t, \xi)|^{2}\right) .
$$

Recalling the notation $v^{2}(\xi)=\left|\sigma_{\mathcal{L}}(\xi)\right|$, this means

$$
\begin{aligned}
& \left|\sigma_{\mathcal{L}}(\xi)\right||\widehat{u}(t, \xi)|^{2}+\left|\partial_{t} \widehat{u}(t, \xi)\right|^{2} \\
& \quad \leq C_{1}^{\prime}\left(\left|\sigma_{\mathcal{L}}(\xi)\right|\left|\widehat{u}_{0}(\xi)\right|^{2}+\left|\widehat{u}_{1}(\xi)\right|^{2}+\sup _{0 \leq t \leq T}|\widehat{f}(t, \xi)|^{2}\right),
\end{aligned}
$$

for all $t \in[0, T], \xi \in \mathcal{I}$, with the constant $C_{1}^{\prime}$ independent of $\xi$. Now we recall that by Plancherel's equality, we have

$$
\begin{aligned}
\left\|\partial_{t} u(t, \cdot)\right\|_{\mathcal{H}}^{2} & =\sum_{\xi \in \mathcal{I}}\left|\partial_{t} \widehat{u}(t, \xi)\right|^{2}, \\
\left\|\mathcal{L}^{1 / 2} u(t, \cdot)\right\|_{\mathcal{H}}^{2} & =\sum_{\xi \in \mathcal{I}}\left|\sigma_{\mathcal{L}}(\xi) \| \widehat{u}(t, \xi)\right|^{2},
\end{aligned}
$$

and

$$
\|f\|_{C([0, T], \mathcal{H})}^{2}=\sum_{\xi \in \mathcal{I}}\|\widehat{f}(\cdot, \xi)\|_{C[0, T]}^{2},
$$

where

$$
\|\widehat{f}(\cdot, \xi)\|_{C[0, T]}^{2}=\sup _{0 \leq t \leq T}|\widehat{f}(t, \xi)|^{2} .
$$

Hence, the estimate (6.10) implies that

$$
\left\|\mathcal{L}^{1 / 2} u(t, \cdot)\right\|_{\mathcal{H}}^{2}+\left\|\partial_{t} u(t, \cdot)\right\|_{\mathcal{H}}^{2} \leq C\left(\left\|\mathcal{L}^{1 / 2} u_{0}\right\|_{\mathcal{H}}^{2}+\left\|u_{1}\right\|_{\mathcal{H}}^{2}+\|f\|_{C([0, T], \mathcal{H})}^{2}\right),
$$

where the constant $C>0$ does not depend on $t \in[0, T]$. More generally, multiplying (6.10) by powers of $\left|\sigma_{\mathcal{L}}(\xi)\right|$, for any $s$, we get

$$
\begin{aligned}
& \left|\sigma_{\mathcal{L}}(\xi)\right|^{1+s}|\widehat{u}(t, \xi)|^{2}+\left|\sigma_{\mathcal{L}}(\xi)\right|^{s}\left|\partial_{t} \widehat{u}(t, \xi)\right|^{2} \\
& \quad \leq C_{1}^{\prime}\left(\left|\sigma_{\mathcal{L}}(\xi)\right|^{1+s}\left|\widehat{u}_{0}(\xi)\right|^{2}+\left|\sigma_{\mathcal{L}}(\xi)\right|^{s}\left|\widehat{u}_{1}(\xi)\right|^{2}+\left|\sigma_{\mathcal{L}}(\xi)\right|^{s} \sup _{0 \leq t \leq T}|\widehat{f}(t, \xi)|^{2}\right) .
\end{aligned}
$$

Taking the sum over $\xi$ as above, this yields the estimate (2.3). 


\subsection{Case I.2: Proof of Theorem 2.2 (a)}

Now, assume that $a(t) \geq a_{0}>0$ but here the regularity of $a$ is less than $C^{1}$, i.e., $a \in C^{\alpha}([0, T])$, with $0<\alpha<1$. Following the notation (6.4) and as in [24] we look for a solution of the system (6.6), i.e. of

$$
\partial_{t} V(t, \xi)=\mathrm{i} v(\xi) A(t, \xi) V(t, \xi)+F(t, \xi),
$$

with

$$
A(t, \xi)=\left(\begin{array}{cc}
0 & 1 \\
a(t) & 0
\end{array}\right)
$$

and

$$
F(t, \xi)=\left(\begin{array}{c}
0 \\
\widehat{f}(t, \xi)
\end{array}\right)
$$

in the form

$$
V(t, \xi)=\mathrm{e}^{-\rho(t) v^{1 / s}(\xi)}(\operatorname{det} H)^{-1} H W,
$$

where $\rho \in C^{1}([0, T])$ is a real-valued function which will be suitably chosen in the sequel, $W=W(t, \xi)$ is to be determined,

$$
H(t)=\left(\begin{array}{cc}
1 & 1 \\
-\lambda(t) & \lambda(t)
\end{array}\right)
$$

and, for $\varphi \in C_{c}^{\infty}(\mathbb{R}), \varphi \geq 0$ with integral 1 ,

$$
\lambda(t, \varepsilon)=\left(\sqrt{a} * \varphi_{\varepsilon}\right)(t),
$$

where

$$
\varphi_{\varepsilon}(t)=\frac{1}{\varepsilon} \varphi(t / \varepsilon) .
$$

By construction, $\lambda$ is smooth in $t \in[0, T]$, and

$$
|\lambda(t, \varepsilon)| \geq \sqrt{a_{0}},
$$

for all $t \in[0, T]$ and $\varepsilon \in(0,1]$,

$$
|\lambda(t, \varepsilon)-\sqrt{a(t)}| \leq C \varepsilon^{\alpha}
$$

uniformly in $t$ and $\varepsilon$. By substitution in (6.13) we get

$$
\begin{aligned}
\mathrm{e}^{-\rho(t) v^{1 / s}(\xi)}(\operatorname{det} H)^{-1} H \partial_{t} W & +\mathrm{e}^{-\rho(t) v^{1 / s}(\xi)}\left(-\rho^{\prime}(t) v^{1 / s}(\xi)\right)(\operatorname{det} H)^{-1} H W \\
& -\mathrm{e}^{-\rho(t) v^{1 / s}(\xi)} \frac{\partial_{t} \operatorname{det} H}{(\operatorname{det} H)^{2}} H W \\
& +\mathrm{e}^{-\rho(t) v^{1 / s}(\xi)}(\operatorname{det} H)^{-1}\left(\partial_{t} H\right) W \\
= & \mathrm{i} v(\xi) \mathrm{e}^{-\rho(t) v^{1 / s}(\xi)}(\operatorname{det} H)^{-1} A H W+F
\end{aligned}
$$


Multiplying both sides of the previous equation by $\mathrm{e}^{\rho(t) v^{1 / s}(\xi)}(\operatorname{det} H) H^{-1}$ we get

$$
\begin{aligned}
\partial_{t} W & -\rho^{\prime}(t) v^{1 / s}(\xi) W-\frac{\partial_{t} \operatorname{det} H}{\operatorname{det} H} W+H^{-1}\left(\partial_{t} H\right) W \\
& =\mathrm{i} v(\xi) H^{-1} A H W+\mathrm{e}^{\rho(t) v^{1 / s}(\xi)}(\operatorname{det} H) H^{-1} F .
\end{aligned}
$$

Hence,

$$
\begin{aligned}
\partial_{t}|W(t, \xi)|^{2}= & 2 \operatorname{Re}\left(\partial_{t} W(t, \xi), W(t, \xi)\right) \\
= & 2 \rho^{\prime}(t) v^{1 / s}(\xi)|W(t, \xi)|^{2} \\
& +2 \frac{\partial_{t} \operatorname{det} H}{\operatorname{det} H}|W(t, \xi)|^{2} \\
& -2 \operatorname{Re}\left(H^{-1} \partial_{t} H W, W\right) \\
& -2 v(\xi) \operatorname{Im}\left(H^{-1} A H W, W\right) \\
& +2 \mathrm{e}^{\rho(t) v^{1 / s}(\xi)} \operatorname{Re}\left((\operatorname{det} H) H^{-1} F, W\right) .
\end{aligned}
$$

It follows that

$$
\begin{aligned}
\partial_{t}|W(t, \xi)|^{2} \leq & 2 \rho^{\prime}(t) v^{1 / s}(\xi)|W(t, \xi)|^{2} \\
& +2\left|\frac{\partial_{t} \operatorname{det} H}{\operatorname{det} H}\right||W(t, \xi)|^{2} \\
& +2\left\|H^{-1} \partial_{t} H\right\|\left|W(t, \xi)^{2}\right| \\
& +v(\xi)\left\|H^{-1} A H-\left(H^{-1} A H\right)^{*}\right\||W(t, \xi)|^{2} \\
& +2 \mathrm{e}^{\rho(t) v^{1 / s}(\xi)}\left\|(\operatorname{det} H) H^{-1}\right\||F \| W|
\end{aligned}
$$

Now we want to show that for all $T>0$ there exist constants $c_{1}, c_{2}>0$ such that

$$
\begin{aligned}
& \left|\frac{\partial_{t} \operatorname{det} H}{\operatorname{det} H}\right| \leq c_{1} \varepsilon^{\alpha-1}, \\
& \left\|H^{-1} \partial_{t} H\right\| \leq c_{2} \varepsilon^{\alpha-1}, \\
& \left\|H^{-1} A H-\left(H^{-1} A H\right)^{*}\right\| \leq c_{3} \varepsilon^{\alpha}, \\
& \left\|(\operatorname{det} H) H^{-1}\right\| \leq c_{4} \varepsilon^{\alpha},
\end{aligned}
$$

for all $t \in[0, T]$ and $\varepsilon \in(0,1]$.

By the definition

$$
\left|\frac{\partial_{t} \operatorname{det} H}{\operatorname{det} H}\right|=\left|\frac{2 \partial_{t}(\lambda(t, \varepsilon))}{2 \lambda(t, \varepsilon)}\right| \leq \frac{1}{\sqrt{a_{0}}}\left|\partial_{t} \lambda(t, \varepsilon)\right| .
$$

For large enough $R$ we have that

$$
\begin{aligned}
& \left|\partial_{t} \lambda(t, \varepsilon)\right|=\varepsilon^{-1}\left|\int_{-R}^{R} \sqrt{a}(\tau) \varphi_{\varepsilon}^{\prime}(t-\tau) d \tau\right|=\left|\varepsilon^{-1} \int_{-R}^{R} \sqrt{a}(t-\varepsilon \tau) \varphi^{\prime}(\tau) d \tau\right| \\
& =\left|\varepsilon^{-1} \int_{-R}^{R}(\sqrt{a}(t-\varepsilon \tau)-\sqrt{a}(t)) \varphi^{\prime}(\tau) d \tau+\varepsilon^{-1} \sqrt{a}(t) \int_{-R}^{R} \varphi^{\prime}(\tau) d \tau\right| \leq C \varepsilon^{-1} \varepsilon^{\alpha}
\end{aligned}
$$


for some constant $C$. From (6.21) and (6.22) we conclude (6.17). Since

$$
\begin{aligned}
\left\|H^{-1} \partial_{t} H\right\| & =\left\|(\lambda(t, \varepsilon)+\lambda(t, \varepsilon))^{-1}\left(\begin{array}{cc}
-\partial_{t} \lambda(t, \varepsilon) & -\partial_{t} \lambda(t, \varepsilon) \\
\partial_{t} \lambda(t, \varepsilon) & \partial_{t} \lambda(t, \varepsilon)
\end{array}\right)\right\| \\
& \leq|2 \lambda(t, \varepsilon)|^{-1}\left(2\left|\partial_{t} \lambda(t, \varepsilon)\right|\right),
\end{aligned}
$$

by using (6.22), we get the estimate (6.18).

Finally, by the direct calculations, we have

$$
\begin{aligned}
\left\|H^{-1} A H-\left(H^{-1} A H\right)^{*}\right\| & =\left\|\frac{1}{\lambda}\left(\begin{array}{cc}
0 & -\left(a-\lambda^{2}\right) \\
a-\lambda^{2} & 0
\end{array}\right)\right\| \\
& =\left|\frac{a-\lambda^{2}}{\lambda}\right| \leq c_{3} \varepsilon^{\alpha},
\end{aligned}
$$

and $\left\|(\operatorname{det} H) H^{-1}\right\| \leq|\lambda| \leq c_{4} \varepsilon^{\alpha}$.

Hence, combining (6.17), (6.18), (6.19) and (6.20) with the energy (6.16) we obtain

$$
\begin{aligned}
\partial_{t}|W(t, \xi)|^{2} \leq & \left(2 \rho^{\prime}(t) v^{1 / s}(\xi)+c_{1} \varepsilon^{\alpha-1}+c_{2} \varepsilon^{\alpha-1}+c_{3} \varepsilon^{\alpha} v(\xi)\right)|W(t, \xi)|^{2} \\
& +c_{4} \mathrm{e}^{(\rho(t)-\mu) v^{1 / s}(\xi)} \varepsilon^{\alpha}|W(t, \xi)|
\end{aligned}
$$

since

$$
|F| \leq C \mathrm{e}^{-\mu v^{1 / s}(\xi)}
$$

for some constants $C, \mu>0$. Without loss of generality, we can assume $v(\xi)>0$. Hence, by setting $\varepsilon:=v^{-1}(\xi)$ we get

$$
\begin{aligned}
\partial_{t}|W(t, \xi)|^{2} \leq\left(2 \rho^{\prime}(t) v^{1 / s}(\xi)+\right. & \left.c_{1}^{\prime} v^{1-\alpha}(\xi)+c_{3}^{\prime} v^{1-\alpha}(\xi)\right)|W(t, \xi)|^{2} \\
& +c_{4} v^{-\alpha}(\xi) \mathrm{e}^{(\rho(t)-\mu) v^{1 / s}(\xi)}|W(t, \xi)| .
\end{aligned}
$$

Set now $\rho(t)=\rho(0)-\kappa t$ with $\rho(0)$ and $\kappa>0$ to be chosen later. Assuming $|W(t, \xi)| \geq 1$ (for the case $|W(t, \xi)| \leq 1$ the same discussions are valid) and taking $\frac{1}{s}>1-\alpha$ we have

$$
\begin{aligned}
\partial_{t}|W(t, \xi)|^{2} \leq & \left(-2 \kappa v^{1 / s}(\xi)+c_{1}^{\prime} v^{1-\alpha}(\xi)+c_{3}^{\prime} v^{1-\alpha}(\xi)\right. \\
& \left.+c_{4} v^{-\alpha}(\xi) \mathrm{e}^{(\rho(0)-\mu) v^{1 / s}(\xi)}\right)|W(t, \xi)|^{2}
\end{aligned}
$$

At this point, setting $\rho(0)<\mu$, for sufficiently large $\nu(\xi)$ we conclude that

$$
\partial_{t}|W(t, \xi)|^{2} \leq 0
$$

for $t \in[0, T]$ and, for example, without loss of generality, for $v(\xi) \geq 1$. Passing now to $V$ we get

$$
\begin{aligned}
|V(t, \xi)| & \leq \mathrm{e}^{-\rho(t) v^{1 / s}(\xi)} \frac{1}{\operatorname{det} H(t)}\|H(t)\||W(t, \xi)| \\
& \leq \mathrm{e}^{-\rho(t) v^{1 / s}(\xi)} \frac{1}{\operatorname{det} H(t)}\|H(t)\||W(0, \xi)| \\
& \leq \mathrm{e}^{(-\rho(t)+\rho(0)) v^{1 / s}(\xi)} \frac{\operatorname{det} H(0)}{\operatorname{det} H(t)}\|H(t)\|\left\|H^{-1}(0)\right\||V(0, \xi)|,
\end{aligned}
$$


where

$$
\frac{\operatorname{det} H(0)}{\operatorname{det} H(t)}\|H(t)\|\left\|H^{-1}(0)\right\| \leq c^{\prime}
$$

This is due to the fact that $\operatorname{det} H(t)$ is a bounded function with

$$
\operatorname{det} H(t)=\lambda_{2}(t)-\lambda_{1}(t) \geq 2 \sqrt{a_{0}}
$$

for all $t \in[0, T]$ and $\varepsilon \in(0,1],\|H(t)\| \leq c$ and $\left\|H^{-1}(0)\right\| \leq c$ for all $t \in[0, T]$ and $\varepsilon \in(0,1]$. Concluding, there exists a constant $c^{\prime}>0$ such that

$$
|V(t, \xi)| \leq c^{\prime} \mathrm{e}^{(-\rho(t)+\rho(0)) v^{1 / s}(\xi)}|V(0, \xi)|
$$

for all $v(\xi) \geq 1$ and $t \in[0, T]$. It is now clear that choosing $\kappa>0$ small enough we have that if $|V(0, \xi)| \leq c \mathrm{e}^{-\delta v^{1 / s}(\xi)}, c, \delta>0$, the same kind of an estimate holds for $V(t, \xi)$. We finally go back to $\xi$ and $\widehat{v}(t, \xi)$. The previous arguments lead to

$$
\begin{aligned}
& v^{2}(\xi)|\widehat{v}(t, \xi)|^{2}+\left|\partial_{t} \widehat{v}(t, \xi)\right|^{2} \\
& \quad \leq c^{\prime} \mathrm{e}^{(-\rho(t)+\rho(0)) v^{1 / s}(\xi)} v^{2}(\xi)\left|\widehat{v_{0}}(\xi)\right|^{2}+c^{\prime} \mathrm{e}^{(-\rho(t)+\rho(0)) v^{1 / s}(\xi)}\left|\widehat{v_{1}}(\xi)\right|^{2}
\end{aligned}
$$

Since the initial data are both in $\gamma_{\mathcal{L}}^{s}$ we obtain that

$$
v^{2}(\xi)|\widehat{v}(t, \xi)|^{2}+\left|\partial_{t} \widehat{v}(t, \xi)\right|^{2} \leq c^{\prime} \mathrm{e}^{\kappa T v^{1 / s}(\xi)}\left(C_{0} \mathrm{e}^{-A_{0} v^{1 / s}(\xi)}+C_{1} \mathrm{e}^{-A_{1} v^{1 / s}(\xi)}\right),
$$

for suitable constants $C_{0}, C_{1}, A_{0}, A_{1}>0$ and $\kappa$ small enough, for $t \in[0, T]$ and all $v(\xi) \geq 1$. The estimate (6.25) implies that under the hypothesis of Case I.2 and for

$$
1 \leq s<1+\frac{\alpha}{1-\alpha}
$$

the solution $u(t, \cdot) \in \gamma_{\mathcal{L}}^{s}$ if the initial data are elements of $\gamma_{\mathcal{L}}^{s}$ and the source term is from $C\left([0, T] ; \gamma_{\mathcal{L}}^{s}\right)$.

\subsection{Case I.2: Proof of Theorem $2.2(b)$}

The proof of Theorem 2.2 (b) follows in an analogous way to deriving the proof of Theorem 2.3 (b) from the proof of Theorem 2.2 (a), and by recalling the characterisation of $H_{(s)}^{-\infty}$ in (5.15). So we refer to the proof of Theorem 2.3 (b) for more details. 


\subsection{Case I.3: Proof of Theorem 2.3 (a)}

We now assume that $a(t) \geq 0$ is of class $C^{\ell}$ on $[0, T]$ with $\ell \geq 2$. Adopting the notations of the previous cases we want to study the well-posedness of the system (6.6): it follows that the equation (6.5) can be written as the first order system

$$
\partial_{t} V(t, \xi)=i v(\xi) A(t) V(t, \xi)+F(t, \xi),
$$

where $V$ is the column vector with entries $V_{1}$ and $V_{2}$,

$$
F(t, \xi)=\left(\begin{array}{c}
0 \\
\widehat{f}(t, \xi)
\end{array}\right)
$$

and

$$
A(t)=\left(\begin{array}{cc}
0 & 1 \\
a(t) & 0
\end{array}\right)
$$

The initial conditions are

$$
V(0, \xi)=\left(\begin{array}{c}
i v(\xi) \widehat{u_{0}}(\xi) \\
\widehat{u}_{1}(\xi)
\end{array}\right)
$$

Let $Q_{\varepsilon}$ be a so-called quasi-symmetriser of $A$, defined by

$$
Q_{\varepsilon}(t):=\left(\begin{array}{cc}
a(t) & 0 \\
0 & 1
\end{array}\right)+\varepsilon^{2}\left(\begin{array}{ll}
1 & 0 \\
0 & 0
\end{array}\right) .
$$

The general technique of using quasi-symmetrisers in weakly hyperbolic problems goes back to D'Ancona and Spagnolo [16]. For its adaptation to the situation similar to the one under our consideration we can also refer to [23].

Now let us introduce the energy

$$
E_{\varepsilon}(t, \xi)=\left(Q_{\varepsilon}(t) V(t, \xi), V(t, \xi)\right) .
$$

By direct computations we get

$\partial_{t} E_{\varepsilon}(t, \xi)=\left(\partial_{t} Q_{\varepsilon} V, V\right)+i v(\xi)\left(\left(Q_{\varepsilon} A-A^{*} Q_{\varepsilon}\right) V, V\right)+\left(Q_{\varepsilon} F, V\right)+\left(Q_{\varepsilon} V, F\right)$

Let us calculate $Q_{\varepsilon} A-A^{*} Q_{\varepsilon}$. By the direct calculations we get

$$
\begin{aligned}
Q_{\varepsilon} A-A^{*} Q_{\varepsilon}= & \left(\begin{array}{cc}
a+\varepsilon^{2} & 0 \\
0 & 1
\end{array}\right)\left(\begin{array}{ll}
0 & 1 \\
a & 0
\end{array}\right) \\
& -\left(\begin{array}{ll}
0 & a \\
1 & 0
\end{array}\right)\left(\begin{array}{cc}
a+\varepsilon^{2} & 0 \\
0 & 1
\end{array}\right)=\left(\begin{array}{cc}
0 & \varepsilon^{2} \\
-\varepsilon^{2} & 0
\end{array}\right) .
\end{aligned}
$$

This implies

$$
\left(\left(Q_{\varepsilon} A-A^{*} Q_{\varepsilon}\right) V, V\right)=\varepsilon^{2} \overline{V_{1}} V_{2}-\varepsilon^{2} V_{1} \overline{V_{2}}
$$


for all $V \in \mathbb{C}^{2}$. By estimating

$$
\begin{aligned}
\left|\left(\left(Q_{\varepsilon} A-A^{*} Q_{\varepsilon}\right) V, V\right)\right| & \leq 2 \varepsilon^{2}\left|V_{1}\right|\left|V_{2}\right| \\
& \leq 2 \varepsilon \sqrt{a+\varepsilon^{2}}\left|V_{1}\right|\left|V_{2}\right| \\
& \leq \varepsilon\left(\left(a+\varepsilon^{2}\right) V_{1}^{2}+V_{2}^{2}\right) \\
& =\varepsilon\left(Q_{\varepsilon} V, V\right),
\end{aligned}
$$

finally, we can write

$$
\left|\left(\left(Q_{\varepsilon} A-A^{*} Q_{\varepsilon}\right) V, V\right)\right| \leq \varepsilon\left(Q_{\varepsilon} V, V\right),
$$

for all $\varepsilon \in(0,1), t \in[0, T]$ and $V \in \mathbb{C}^{2}$.

Now to estimate (6.26) we prove first that there exists a constant $C \geq 1$ such that

$$
C^{-1} \varepsilon^{2}|V|^{2} \leq\left(Q_{\varepsilon} V, V\right) \leq C|V|^{2},
$$

for all $\varepsilon \in(0,1], t \in[0, T]$ and all non-zero continuous functions $V:[0, T] \times \mathcal{I} \rightarrow$ $\mathbb{C}^{2}$.

By recalling components of $V=\left(V_{1}, V_{2}\right)$, we have

$$
\left(Q_{\varepsilon} V, V\right)=\left(a+\varepsilon^{2}\right) V_{1}^{2}+V_{2}^{2} \leq C\left(V_{1}^{2}+V_{2}^{2}\right)=C|V|^{2},
$$

and

$\left(Q_{\varepsilon} V, V\right)=\left(a+\varepsilon^{2}\right) V_{1}^{2}+V_{2}^{2} \geq \varepsilon^{2} V_{1}^{2}+V_{2}^{2} \geq C^{-1}\left(\varepsilon^{2} V_{1}^{2}+\varepsilon^{2} V_{2}^{2}\right)=C^{-1} \varepsilon^{2}|V|^{2}$

for some constant $C \geq 1$.

Note, that for all $\varepsilon \in(0,1], t \in[0, T]$ and all non-zero continuous functions $V:[0, T] \times \mathcal{I} \rightarrow \mathbb{C}^{2}$, we get

$$
\int_{0}^{T} \frac{\left|\left(\partial_{t} Q_{\varepsilon}(t) V(t, \xi), V(t, \xi)\right)\right|}{\left(Q_{\varepsilon}(t) V(t, \xi), V(t, \xi)\right)} d t \leq C \varepsilon^{-2 / \ell},
$$

for some $C_{1}>0$. For more details on the estimate (6.30), see [23], or [36].

Since $f \in C\left([0, T] ; \gamma_{\mathcal{L}}^{s}\right)$, we have

$$
|\widehat{f}(t, \xi)| \leq C \mathrm{e}^{-\mu \nu^{1 / s}(\xi)}
$$

for all $t \in[0, T]$ and $\xi$, and for some constants $C, \mu>0$, we obtain

$$
\left|\left(Q_{\varepsilon} F, V\right)+\left(Q_{\varepsilon} V, F\right)\right| \leq 2\left\|Q_{\varepsilon}\right\||F||V| \leq C_{1} \mathrm{e}^{-\mu \nu^{1 / s}(\xi)}|V|
$$

for some constant $C_{1}$. Assuming $|V| \geq 1(|V| \leq 1$ can be considered in a similar way) and by using (6.28) and (6.30) in (6.26), and by Gronwall's lemma, we get

$$
E_{\varepsilon}(t, \xi) \leq E_{\varepsilon}(0, \xi) \mathrm{e}^{c\left(\varepsilon^{-2 / \ell}+\varepsilon v(\xi)\right)}
$$

for some constant $c>0$, uniformly in $t, \xi$ and $\varepsilon$. By setting

$$
\varepsilon^{-2 / \ell}=\varepsilon v(\xi)
$$


we arrive at

$$
E_{\varepsilon}(t, \xi) \leq E_{\varepsilon}(0, \xi) C_{T} \mathrm{e}^{C_{T} v^{\frac{1}{\sigma}}(\xi)},
$$

with $\sigma=1+\frac{\ell}{2}$. An application of (6.29) yields the estimate

$$
C^{-1} \varepsilon^{2}|V(t, \xi)|^{2} \leq E_{\varepsilon}(t, \xi) \leq E_{\varepsilon}(0, \xi) C_{T} \mathrm{e}^{C_{T} v^{\frac{1}{\sigma}}(\xi)} \leq C|V(0, \xi)|^{2} C_{T} \mathrm{e}^{C_{T} v^{\frac{1}{\sigma}}(\xi)}
$$

which implies

$$
|V(t, \xi)| \leq C_{2} v^{\frac{\ell}{2 \sigma}}(\xi) \mathrm{e}^{C v^{\frac{1}{\sigma}}(\xi)}|V(0, \xi)|,
$$

for some $C_{2}>0$, for all $t \in[0, T]$ and for all $\xi$. We now go back to $\widehat{u}(t, \xi)$. Hence, we get

$$
|\widehat{u}(t, \xi)|^{2} \leq C^{2} v^{\frac{\ell}{\sigma}}(\xi) \mathrm{e}^{2 C v^{\frac{1}{\sigma}}(\xi)}\left(v^{2}(\xi)\left|\widehat{u}_{0}(\xi)\right|^{2}+\left|\widehat{u}_{1}(\xi)\right|^{2}\right) .
$$

Recall that the initial data $u_{0}$ and $u_{1}$ are elements of $\gamma_{\mathcal{L}}^{s}$ and, therefore, there exist constants $A^{\prime}, C^{\prime}>0$ such that

$$
\left|\mathrm{e}^{A^{\prime}\left|\sigma_{\mathcal{L}}(\xi)\right| \frac{1}{2 s}} \widehat{u}_{0}(\xi)\right| \leq C^{\prime}, \quad\left|\mathrm{e}^{A^{\prime}\left|\sigma_{\mathcal{L}}(\xi)\right|^{\frac{1}{2 s}}} \widehat{u}_{1}(\xi)\right| \leq C^{\prime} .
$$

Inserting (6.33) in (6.32), taking $s<\sigma$ and $v(\xi)$ large enough we conclude that there exist constants $C^{\prime \prime}>0$ such that

$$
\left|\mathrm{e}^{A^{\prime}\left|\sigma_{\mathcal{L}}(\xi)\right|^{\frac{1}{2 s}}} \widehat{u}(t, \xi)\right|^{2} \leq C^{\prime \prime},
$$

for all $t \in[0, T]$. It follows that

$$
\sum_{\xi \in \mathcal{I}}\left|\mathrm{e}^{\frac{A^{\prime}}{2}\left|\sigma_{\mathcal{L}}(\xi)\right|^{\frac{1}{2 s}}} \widehat{u}(t, \xi)\right|^{2}<\infty,
$$

i.e. $u(t, \cdot) \in \gamma_{\mathcal{L}}^{S}$ provided that

$$
1 \leq s<\sigma=1+\frac{\ell}{2}
$$

\subsection{Case I.3: Proof of Theorem $2.3(b)$}

Not changing anything in the proof of Theorem 2.3 (a), analogously, in this case we have estimate (6.32). Recall the characterisation of $H_{(s)}^{-\infty}$. Since $u_{0}$ and $u_{1}$ are elements of $H_{(s)}^{-\infty}, f \in C\left([0, T] ; H_{(s)}^{-\infty}\right)$, and, therefore, by Corollary 5.2 there exist constants $A_{1}, C_{1}>0$ such that

$$
\begin{aligned}
\left|\mathrm{e}^{-A_{1}\left|\sigma_{\mathcal{L}}(\xi)\right| \frac{1}{2 s}} \widehat{u}_{0}(\xi)\right| \leq C_{1}, \\
\left|\mathrm{e}^{-A_{1}\left|\sigma_{\mathcal{L}}(\xi)\right|^{\frac{1}{2 s}}} \widehat{u}_{1}(\xi)\right| \leq C_{1}, \\
\sup _{t \in[0, T]}\left|\mathrm{e}^{-A_{1}\left|\sigma_{\mathcal{L}}(\xi)\right|^{\frac{1}{2 s}}} \widehat{f}(t, \xi)\right| \leq C_{1} .
\end{aligned}
$$


Inserting (6.34) in (6.32), taking $s<\sigma$ and $v(\xi)$ large enough we conclude that there exist constants $C_{2}, A_{2}>0$ such that

$$
\left|\mathrm{e}^{-A_{2}\left|\sigma_{\mathcal{L}}(\xi)\right| \frac{1}{2 s}} \widehat{u}(t, \xi)\right|^{2} \leq C_{2},
$$

for all $t \in[0, T]$. It follows that there are constants $A, C>0$ such that

$$
|\widehat{u}(t, \xi)| \leq C \mathrm{e}^{A v^{\frac{1}{s}}(\xi)},
$$

i.e. $u(t, \cdot) \in H_{(s)}^{-\infty}$ provided that

$$
1 \leq s<\sigma=1+\frac{\ell}{2}
$$

This completes the proof of Theorem 2.3 (b).

\subsection{Case I.4: Proof of Theorem 2.4 (a)}

Now assume $a(t) \geq 0$ and $a \in C^{\alpha}([0, T])$ with $0<\alpha<2$. Here the roots $\pm \sqrt{a(t)}$ can coincide and are not Hölder of order $\alpha$ but of order $\alpha / 2$. For an adaptation of the proof of Theorem 2.2 we will set that $a \in C^{2 \alpha}([0, T]), 0<\alpha<1$, and that the roots are from $C^{\alpha}$. Again we seek a solution of the system (6.13) in the form

$$
V(t, \xi)=\mathrm{e}^{-\rho(t) v^{\frac{1}{s}}(\xi)}(\operatorname{det} H)^{-1} H W,
$$

where $\rho \in C^{1}([0, T])$ is a real valued function which will be suitably chosen in the sequel,

$$
H(t)=\left(\begin{array}{cc}
1 & 1 \\
\lambda_{1}(t, \varepsilon) & \lambda_{2}(t, \varepsilon)
\end{array}\right)
$$

and, for $\varphi \in C_{0}^{\infty}(\mathbb{R}), \varphi \geq 0$ with integral 1 , we set

$$
\begin{aligned}
& \lambda_{1}(t, \varepsilon)=\left(-\sqrt{a} * \varphi_{\varepsilon}\right)(t)+\varepsilon^{\alpha}, \\
& \lambda_{2}(t, \varepsilon)=\left(+\sqrt{a} * \varphi_{\varepsilon}\right)(t)+2 \varepsilon^{\alpha} .
\end{aligned}
$$

Note that $\lambda_{1}$ and $\lambda_{2}$ are smooth in $t \in[0, T]$, and

$$
\lambda_{2}(t, \varepsilon)-\lambda_{1}(t, \varepsilon) \geq \varepsilon^{\alpha},
$$

for all $t \in[0, T]$ and $\varepsilon \in(0,1]$,

$$
\left|\lambda_{1}(t, \varepsilon)+\sqrt{a(t)}\right| \leq c_{1} \varepsilon^{\alpha}
$$

and

$$
\left|\lambda_{2}(t, \varepsilon)-\sqrt{a(t)}\right| \leq c_{2} \varepsilon^{\alpha},
$$


uniformly in $t$ and $\varepsilon$. In analogy to the Case I.2 we take the energy estimate

$$
\begin{aligned}
\partial_{t}|W(t, \xi)|^{2} \leq & 2 \rho^{\prime}(t) v^{\frac{1}{s}}(\xi)|W(t, \xi)|^{2}+2\left|\frac{\partial_{t} \operatorname{det} H}{\operatorname{det} H}\right||W(t, \xi)|^{2} \\
& +\left.2\left\|H^{-1} \partial_{t} H\right\|\left|W(t, \xi)^{2}+v(\xi)\left\|H^{-1} A H-\left(H^{-1} A H\right)^{*}\right\|\right| W(t, \xi)\right|^{2} \\
& +2 \mathrm{e}^{\rho(t) v^{1 / s}(\xi)}\left\|(\operatorname{det} H) H^{-1}\right\||F \| W| .
\end{aligned}
$$

By using (6.22), (6.36) and discussions of the proof of the Case I.2, it is easy to show that for all $T>0$ there exist constants $c_{1}, c_{2}>0$ such that

$$
\begin{aligned}
& \left|\frac{\partial_{t} \operatorname{det} H}{\operatorname{det} H}\right| \leq c_{1} \varepsilon^{1}, \\
& \left\|H^{-1} \partial_{t} H\right\| \leq c_{2} \varepsilon^{-1},
\end{aligned}
$$

for all $t \in[0, T]$ and $\varepsilon \in(0,1]$.

Now, let us show that

$$
\left\|H^{-1} A H-\left(H^{-1} A H\right)^{*}\right\| \leq c \varepsilon^{\alpha},
$$

for some $c$, and for all $t \in[0, T]$ and $\varepsilon \in(0,1]$. By the simple calculations we get

$$
\begin{aligned}
\left\|H^{-1} A H-\left(H^{-1} A H\right)^{*}\right\| & =\left\|\frac{1}{\lambda_{2}-\lambda_{1}}\left(\begin{array}{cc}
0 & \left(\lambda_{1}^{2}+\lambda_{2}^{2}-2 a\right) \\
-\left(\lambda_{1}^{2}+\lambda_{2}^{2}-2 a\right) & 0
\end{array}\right)\right\| \\
& \leq \varepsilon^{-\alpha}\left|\lambda_{1}^{2}+\lambda_{2}^{2}-2 a\right| \\
& =\varepsilon^{-\alpha}\left|\left(\lambda_{1}-\sqrt{a}\right)\left(\lambda_{1}+\sqrt{a}\right)+\left(\lambda_{2}-\sqrt{a}\right)\left(\lambda_{2}+\sqrt{a}\right)\right| \\
& \leq C\left(\left|\left(\lambda_{1}-\sqrt{a}\right)\right|+\left|\left(\lambda_{2}+\sqrt{a}\right)\right|\right) .
\end{aligned}
$$

Indeed, there is a sufficiently large $R$ and constants $C_{1}, C_{2}$ such that

$$
\begin{aligned}
\left|\lambda_{1}(t, \varepsilon)-\sqrt{a}\right| & =\left|\int_{-R}^{R}(\sqrt{a}(t-\varepsilon \tau)-\sqrt{a}(t)) \varphi^{\prime}(\tau) d \tau+\varepsilon^{2}\right| \leq C_{1} \varepsilon^{\alpha}, \\
\left|\lambda_{2}(t, \varepsilon)+\sqrt{a}\right| & =\left|\int_{-R}^{R}(\sqrt{a}(t-\varepsilon \tau)-\sqrt{a}(t)) \varphi^{\prime}(\tau) d \tau+2 \varepsilon^{2}\right| \leq C_{2} \varepsilon^{\alpha} .
\end{aligned}
$$

Then (6.40) holds.

By combining (6.38), (6.39) and (6.40) for $|W(t, \xi)|^{2}$ we obtain

$$
\begin{aligned}
\partial_{t}|W(t, \xi)|^{2} \leq & \left(2 \rho^{\prime}(t) v^{\frac{1}{s}}(\xi)+c_{1} \varepsilon^{-1}+c_{2} \varepsilon^{-1}+c_{3} \varepsilon^{\alpha} v(\xi)\right)|W(t, \xi)|^{2} \\
& +c_{4} \mathrm{e}^{(\rho(t)-\mu) v^{1 / s}(\xi)} \varepsilon^{\alpha}|W(t, \xi)|
\end{aligned}
$$

for some constants $c_{4}$ and $\mu>0$.

Consider the case $|W(t, \xi)| \geq 1$. Again, it is not restrictive to assume that $\nu(\xi)>0$. Setting

$$
\varepsilon:=v^{-\gamma}(\xi)
$$


with

$$
\gamma=\frac{1}{1+\alpha}
$$

we get

$$
\begin{aligned}
\partial_{t}|W(t, \xi)|^{2} \leq\left(2 \rho^{\prime}(t) v^{\frac{1}{s}}(\xi)+c_{1}^{\prime} v^{\gamma}(\xi)+c_{3}^{\prime} v^{1-\gamma \alpha}(\xi)\right. \\
\left.\quad+c_{4}^{\prime} \mathrm{e}^{(\rho(t)-\mu) v^{1 / s}(\xi)} v^{-\gamma \alpha}(\xi)\right)|W(t, \xi)|^{2} \\
\left.\leq\left(2 \rho^{\prime}(t) v^{\frac{1}{s}}(\xi)+C_{1} v^{1 /(1+\alpha)}(\xi)+C_{2} \mathrm{e}^{(\rho(t)-\mu) v^{1 / s}(\xi)} v^{-\frac{\alpha}{1+\alpha}}\right)(\xi)\right)|W(t, \xi)|^{2} .
\end{aligned}
$$

At this point taking

$$
\frac{1}{s}>\frac{1}{1+\alpha}
$$

and

$$
\rho(t)=\rho(0)-\kappa t
$$

with $\kappa>0$ to be chosen later, for large enough $\nu(\xi)$ and $\rho(0)<\mu$ we conclude that

$$
\partial_{t}|W(t, \xi)|^{2} \leq 0
$$

for $t \in[0, T]$ and $v(\xi) \geq 1$. Passing now to $V$ and by the same arguments of Case I. 2 with

$$
\frac{\operatorname{det} H(0)}{\operatorname{det} H(t)}\|H(t)\|\left\|H^{-1}(0)\right\| \leq c \varepsilon^{-\alpha}=c v^{\gamma \alpha}(\xi)=c v^{\frac{\alpha}{1+\alpha}}(\xi)
$$

we conclude that there exists a constant $c^{\prime}>0$ such that

$$
|V(t, \xi)| \leq c^{\prime} v^{\frac{\alpha}{1+\alpha}}(\xi) \mathrm{e}^{(-\rho(t)+\rho(0)) v^{\frac{1}{s}}(\xi)}|V(0, \xi)|,
$$

for all $v(\xi) \geq 1$ and $t \in[0, T]$. We finally go back to $\widehat{u}(t, \xi)$. We have

$$
v^{2}(\xi)|\widehat{u}(t, \xi)|^{2} \leq c^{\prime} \mathrm{e}^{(-\rho(t)+\rho(0)) \nu(\xi)^{\frac{1}{s}}}\left(v(\xi)^{2}\left|\widehat{u}_{0}(\xi)\right|^{2}+\left|\widehat{u}_{1}(\xi)\right|^{2}\right),
$$

with the constant $c^{\prime}$ independent of $\xi$. Multiplying by $\mathrm{e}^{\delta v(\xi)^{\frac{1}{s}}}$, we get

$$
\begin{aligned}
& \left|\mathrm{e}^{\delta\left|\sigma_{\mathcal{L}}(\xi)\right|^{\frac{1}{2 s}}} \sigma_{\mathcal{L}}(\xi) \widehat{u}(t, \xi)\right|^{2} \\
& \quad \leq c^{\prime}\left(\left|\mathrm{e}^{(-\rho(t)+\rho(0)+\delta)\left|\sigma_{\mathcal{L}}(\xi)\right|^{\frac{1}{2 s}}} \sigma_{\mathcal{L}}(\xi) \widehat{u}_{0}(\xi)\right|^{2}+\left|\mathrm{e}^{(-\rho(t)+\rho(0)+\delta)\left|\sigma_{\mathcal{L}}(\xi)\right|^{\frac{1}{2 s}}} \widehat{u}_{1}(\xi)\right|^{2}\right),
\end{aligned}
$$

for any $\delta>0$. Since the initial data are both in $\gamma_{\mathcal{L}}^{s}$, we get that

$$
\sum_{\xi \in \mathcal{I}}\left(\left|\mathrm{e}^{(\kappa T+\delta)\left|\sigma_{\mathcal{L}}(\xi)\right|^{\frac{1}{2 s}}} \sigma_{\mathcal{L}}(\xi) \widehat{u}_{0}(\xi)\right|^{2}+\left|\mathrm{e}^{(\kappa T+\delta)\left|\sigma_{\mathcal{L}}(\xi)\right| \frac{1}{2 s}} \widehat{u}_{1}(\xi)\right|^{2}\right)<\infty
$$


for some $\delta>0$ if $\kappa$ is small enough. Taking the same sum $\sum_{\xi \in \mathcal{I}}$ of the expressions in (6.43), and using Lemma 5.1, we obtain that

$$
\sum_{\xi \in \mathcal{I}}\left|\mathrm{e}^{\delta\left|\sigma_{\mathcal{L}}(\xi)\right|^{\frac{1}{2 s}}} \sigma_{\mathcal{L}}(\xi) \widehat{u}(t, \xi)\right|^{2}<\infty,
$$

for $\kappa$ small enough, for $t \in[0, T]$. This completes the proof of Theorem 2.4.

\subsection{Case I.4: Proof of Theorem $2.4(b)$}

Similarly to the proof of Theorem 2.3 (b) following from the proof of Theorem 2.4 (a), recalling the characterisation of $H_{(s)}^{-\infty}$, we get the proof of Theorem 2.4 (b).

\section{Proofs of Part II}

We start by proving Theorem 4.3 assuring the existence of very weak solutions.

\subsection{Existence of Very Weak Solutions}

As in Theorem 4.3 we consider two cases.

Case II.1. We now assume that coefficient $a=a(t)$ is a distribution with compact support contained in $[0, T]$. Since the formulation of (4.3) in this case might be impossible in the distributional sense due to issues related to the product of distributions, we replace (4.3) with a regularised equation. In other words, we regularise $a$ by a convolution with a mollifier in $C_{0}^{\infty}(\mathbb{R})$ and get nets of smooth functions as coefficients. More precisely, let $\psi \in C_{0}^{\infty}(\mathbb{R}), \psi \geq 0$ with $\int \psi=1$, and let $\omega(\varepsilon)$ be a positive function converging to 0 as $\varepsilon \rightarrow 0$, with the rate of convergence to be specified later. Define

$$
\begin{aligned}
\psi_{\omega(\varepsilon)}(t) & :=\frac{1}{\omega(\varepsilon)} \psi\left(\frac{t}{\omega(\varepsilon)}\right), \\
a_{\varepsilon}(t) & :=\left(a * \psi_{\omega(\varepsilon)}\right)(t), \quad f_{\varepsilon}(t):=\left(f(\cdot) * \psi_{\omega(\varepsilon)}\right)(t), \quad t \in[0, T] .
\end{aligned}
$$

Since $a$ is a positive distribution with compact support (hence a Radon measure) and $\psi \in C_{0}^{\infty}(\mathbb{R})$, supp $\psi \subset K, \psi \geq 0$, identifying the measure $a$ with its density, we can write

$$
\begin{aligned}
a_{\varepsilon}(t) & =\left(a * \psi_{\omega(\varepsilon)}\right)(t)=\int_{\mathbb{R}} a(t-\tau) \psi_{\omega(\varepsilon)}(\tau) d \tau=\int_{\mathbb{R}} a(t-\omega(\varepsilon) \tau) \psi(\tau) d \tau \\
& =\int_{\mathrm{K}} a(t-\omega(\varepsilon) \tau) \psi(\tau) d \tau \geq a_{0} \int_{\mathrm{K}} \psi(\tau) d \tau:=\tilde{a}_{0}>0,
\end{aligned}
$$

with a positive constant $\tilde{a}_{0}>0$ independent of $\varepsilon$. 
By the structure theorem for compactly supported distributions, we have that there exist $L_{1}, L_{2} \in \mathbb{N}$ and $c_{1}, c_{2}>0$ such that

$$
\left|\partial_{t}^{k} a_{\varepsilon}(t)\right| \leq c_{1} \omega(\varepsilon)^{-L_{1}-k}, \quad\left|\partial_{t}^{k} f_{\varepsilon}(t)\right| \leq c_{2} \omega(\varepsilon)^{-L_{2}-k},
$$

for all $k \in \mathbb{N}_{0}$ and $t \in[0, T]$. We note that the numbers $L_{1}$ and $L_{2}$ may be related to the distributional orders of $a$ and $f$ but we will not be needing such a relation in our proof.

Hence, $a_{\varepsilon}$ and $f_{\varepsilon}$ are $C^{\infty}$-moderate regularisations of the coefficient $a$ and of the source term $f$. Now, fix $\varepsilon \in(0,1]$, and consider the regularised problem

$$
\left\{\begin{aligned}
\partial_{t}^{2} u_{\varepsilon}(t)+a_{\varepsilon}(t) \mathcal{L} u_{\varepsilon}(t) & =f_{\varepsilon}(t), t \in[0, T], \\
u_{\varepsilon}(0) & =u_{0} \in \mathcal{H}, \\
\partial_{t} u_{\varepsilon}(0) & =u_{1} \in \mathcal{H},
\end{aligned}\right.
$$

with the Cauchy data satisfying $\left(u_{0}, u_{1}\right) \in H_{\mathcal{L}}^{s+1} \times H_{\mathcal{L}}^{s}, a_{\varepsilon} \in C^{\infty}[0, T]$ and also $f_{\varepsilon} \in C^{\infty}\left([0, T] ; H_{\mathcal{L}}^{s}\right)$. Then all discussions and calculations of Theorem 2.1 are valid. Thus by Theorem 2.1 the equation (7.2) has a unique solution in the space $C\left([0, T] ; H_{\mathcal{L}}^{s+1}\right) \cap C^{1}\left([0, T] ; H_{\mathcal{L}}^{s}\right)$. In fact, this unique solution is from $C^{\infty}\left([0, T] ; H_{\mathcal{L}}^{s}\right)$. This can be checked by taking in account that $a_{\varepsilon} \in C^{\infty}([0, T])$ and by differentiating both sides of the equation (7.2) in $t$ inductively. Applying Theorem 2.1 to the equation (7.2), using the inequality

$$
\left\|\partial_{t} S(t, \xi)\right\| \leq C\left|\partial_{t} a_{\varepsilon}(t)\right| \leq C \omega(\varepsilon)^{-L-1}
$$

and Gronwall's lemma, we get the estimate

$$
\begin{aligned}
& \left\|u_{\varepsilon}(t, \cdot)\right\|_{H_{\mathcal{L}}^{s+1}}^{2}+\left\|\partial_{t} u_{\varepsilon}(t, \cdot)\right\|_{H_{\mathcal{L}}^{s}}^{2} \\
& \quad \leq C \exp \left(c \omega(\varepsilon)^{-L-1} T\right)\left(\left\|u_{0}\right\|_{H_{\mathcal{L}}^{s+1}}^{2}+\left\|u_{1}\right\|_{H_{\mathcal{L}}^{s}}^{2}+\|f\|_{C\left([0, T] ; H_{\mathcal{L}}^{s}\right)}^{2}\right)
\end{aligned}
$$

where the coefficient $L$ is deduced from (7.1).

Put $\omega^{-L-1}(\varepsilon) \sim \log \varepsilon$. Then the estimate (7.3) transforms to

$$
\left\|u_{\varepsilon}(t, \cdot)\right\|_{H_{\mathcal{L}}^{s+1}}^{2}+\left\|\partial_{t} u_{\varepsilon}(t, \cdot)\right\|_{H_{\mathcal{L}}^{s}}^{2} \leq C \varepsilon^{-L-1}\left(\left\|u_{0}\right\|_{H_{\mathcal{L}}^{s+1}}^{2}+\left\|u_{1}\right\|_{H_{\mathcal{L}}^{s}}^{2}+\|f\|_{C\left([0, T] ; H_{\mathcal{L}}^{s}\right)}^{2}\right),
$$

with possibly new constant $L$. To simplify the notation we continue denoting them by the same letters.

Now, let us show that there exist $N \in \mathbb{N}_{0}, c>0$ and, for all $k \in \mathbb{N}_{0}$ there exist $N_{k}>0$ and $c_{k}>0$ such that

$$
\left\|\partial_{t}^{k} u_{\varepsilon}(t, \cdot)\right\|_{H_{\mathcal{L}}^{s}} \leq c_{k} \varepsilon^{-N-k}
$$

for all $t \in[0, T]$, and $\varepsilon \in(0,1]$.

Applying (6.8) and (6.9) to $u_{\varepsilon}$, and by taking account the properties of $a_{\varepsilon}$, we get

$$
\begin{aligned}
\left|\sigma_{\mathcal{L}}(\xi)\right|\left|\widehat{u_{\varepsilon}}(t, \xi)\right|^{2} & +\left|\partial_{t} \widehat{u_{\varepsilon}}(t, \xi)\right|^{2} \\
& \leq C \varepsilon^{-L-1}\left(\left|\sigma_{\mathcal{L}}(\xi)\right|\left|\widehat{u}_{0}(\xi)\right|^{2}+\left|\widehat{u}_{1}(\xi)\right|^{2}+\sup _{t \in[0, T]}|\widehat{f}(t, \xi)|^{2}\right)
\end{aligned}
$$


for all $t \in[0, T], \xi \in \mathcal{I}$, for some $L>0$ with the constant $C$ independent of $\xi$. Thus, multiplying this by appropriate powers of $\left|\sigma_{\mathcal{L}}(\xi)\right|$ we obtain

$$
\left\|\partial_{t} u_{\varepsilon}(t, \cdot)\right\|_{H_{\mathcal{L}}^{s}} \leq C \varepsilon^{-L-1}, \quad\left\|u_{\varepsilon}(t, \cdot)\right\|_{H_{\mathcal{L}}^{s+1}} \leq C \varepsilon^{-L} .
$$

Acting by the iterations of $\partial_{t}$ on the equality

$$
\partial_{t}^{2} u_{\varepsilon}(t)=-a_{\varepsilon}(t) \mathcal{L} u_{\varepsilon}(t)+f_{\varepsilon}(t)
$$

and taking it in $\mathcal{H}$-norms, we conclude that $u_{\varepsilon}$ is $C^{\infty}\left([0, T] ; H_{\mathcal{L}}^{S}\right)$-moderate.

This shows that the Cauchy problem (4.3) has a very weak solution.

Case II.2. Repeating discussions of Case II.1, in this case we get that for a nonnegative function $a_{\varepsilon}(t)$ there exist $L \in \mathbb{N}$ and $c_{1}>0$ such that

$$
\left|\partial_{t}^{k} a_{\varepsilon}(t)\right| \leq c_{1} \omega(\varepsilon)^{-L-k},
$$

for all $k \in \mathbb{N}_{0}$ and $t \in[0, T]$, i.e. $a_{\varepsilon}$ and $f_{\varepsilon}$ are $C^{\infty}$-moderate regularisations of the coefficient $a$ and of the source term $f$. Fix $\varepsilon \in(0,1]$, and consider the regularised problem

$$
\left\{\begin{aligned}
\partial_{t}^{2} u_{\varepsilon}(t)+a_{\varepsilon}(t) \mathcal{L} u_{\varepsilon}(t) & =f_{\varepsilon}(t), t \in[0, T], \\
u_{\varepsilon}(0) & =u_{0} \in \mathcal{H}, \\
\partial_{t} u_{\varepsilon}(0) & =u_{1} \in \mathcal{H},
\end{aligned}\right.
$$

with the Cauchy data satisfy $u_{0}, u_{1} \in H_{(s)}^{-\infty}$ and $a_{\varepsilon} \in C^{\infty}[0, T]$. Then all discussions and calculations of Theorem 2.3 (b) are valid. Thus by Theorem 2.3 (b) the equation (7.5) has a unique solution in the space $u \in C^{2}\left([0, T] ; H_{(s)}^{-\infty}\right)$ for any $s$. In fact, this unique solution is from $C^{\infty}\left([0, T] ; H_{(s)}^{-\infty}\right)$. This can be checked by taking in account that $a_{\varepsilon} \in C^{\infty}([0, T])$ and by differentiating both sides of the equation (7.5) in $t$ inductively. Applying Theorem 2.3 (b) to the equation (7.5), using the inequality

$$
\left|\partial_{t} a_{\varepsilon}(t)\right| \leq C \omega(\varepsilon)^{-L-1},
$$

we get the estimate

$$
\begin{aligned}
\left|\widehat{u_{\varepsilon}}(t, \xi)\right|^{2}+\left|\partial_{t} \widehat{u_{\varepsilon}}(t, \xi)\right|^{2} \leq & \leq C^{2} v^{\frac{\ell}{\sigma}}(\xi) \mathrm{e}^{2 C \omega(\varepsilon)^{-L-1} v^{\frac{1}{\sigma}}(\xi)}\left(v^{2}(\xi)\left|\widehat{u}_{0, \varepsilon}(\xi)\right|^{2}\right. \\
& \left.+\left|\widehat{u}_{1, \varepsilon}(\xi)\right|^{2}+\sup _{t \in[0, T]}|\widehat{f}(t, \xi)|^{2}\right)
\end{aligned}
$$

By putting $\omega^{-1}(\varepsilon) \sim(\log \varepsilon)^{r}$ for an appropriate $r$, and repeating as in the proof of Theorem 2.3 (b), from (7.6) we conclude (a similar argument is also in [25]) that there exists $\eta>0$ and, for $p=0,1$ there exist $c_{p}>0$ and $N_{p}>0$ such that

$$
\left\|\mathrm{e}^{-\eta \mathcal{L}^{\frac{1}{2 s}}} \partial_{t}^{p} u_{\varepsilon}(t, \cdot)\right\|_{\mathcal{H}} \leq c_{p} \varepsilon^{-N_{p}-p}
$$

for all $t \in[0, T]$ and $\varepsilon \in(0,1]$. Now, we need to prove that the estimate (7.7) holds for all $p \in \mathbb{N}$. To show this we use the equality

$$
\partial_{t}^{2} u_{\varepsilon}(t)=-a_{\varepsilon}(t) \mathcal{L} u_{\varepsilon}(t)+f_{\varepsilon}(t)
$$

Acting by the iterations of $\partial_{t}$ on the last equality and using properties of $a_{\varepsilon}$ and the estimate (7.7), we obtain that $u_{\varepsilon}$ is $C^{\infty}\left([0, T] ; H_{(s)}^{-\infty}\right)$-moderate.

Theorem 4.3 is proved. 


\subsection{Consistency with the Classical Well-Posedness}

Here we show that when the coefficients are regular enough then the very weak solution coincides with the classical one: this is the content of Theorem 4.4 which we will prove here.

Moreover, we show that the very weak solution provided by Theorem 4.3 is unique in an appropriate sense. For the formulation of the uniqueness statement it will be convenient to use the language of Colombeau algebras.

Definition 7.1. We say that $\left(u_{\varepsilon}\right)_{\varepsilon}$ is $C^{\infty}$-negligible if for all $K \Subset \mathbb{R}$, for all $\alpha \in \mathbb{N}$ and for all $\ell \in \mathbb{N}$ there exists a constant $c>0$ such that

$$
\sup _{t \in K}\left|\partial^{\alpha} u_{\varepsilon}(t)\right| \leq c \varepsilon^{\ell}
$$

for all $\varepsilon \in(0,1]$.

Since we are dealing with time-dependent distributions supported in the interval $[0, T]$, it is sufficient to take $K=[0, T]$ in the above definition.

We now introduce the Colombeau algebra as the quotient

$$
\mathcal{G}(\mathbb{R})=\frac{C^{\infty}-\text { moderate nets }}{C^{\infty}-\text { negligible nets }} .
$$

For the general analysis of $\mathcal{G}(\mathbb{R})$ we refer to e.g. Oberguggenberger [45].

Theorem 7.2. (Uniqueness)

(II.1) Let a be a positive distribution with compact support included in [0,T], such that $a \geq a_{0}$ for some constant $a_{0}>0$. Let $\left(u_{0}, u_{1}\right) \in H_{\mathcal{L}}^{s+1} \times H_{\mathcal{L}}^{s}$ and $f \in \mathcal{G}\left([0, T] ; H_{\mathcal{L}}^{s}\right)$ for some $s \in \mathbb{R}$. Then there exists an embedding of the coefficient a into $\mathcal{G}([0, T])$, such that the Cauchy problem (4.3), that is

$$
\left\{\begin{aligned}
\partial_{t}^{2} u(t)+a(t) \mathcal{L} u(t) & =f(t), t \in[0, T], \\
u(0) & =u_{0} \in \mathcal{H}, \\
\partial_{t} u(0) & =u_{1} \in \mathcal{H},
\end{aligned}\right.
$$

has a unique solution $u \in \mathcal{G}\left([0, T] ; H_{\mathcal{L}}^{s}\right)$.

(II.2) Let $a \geq 0$ be a nonnegative distribution with compact support included in $[0, T]$. Let $u_{0}, u_{1} \in H_{(s)}^{-\infty}$ and $f \in \mathcal{G}\left([0, T] ; H_{(s)}^{-\infty}\right)$ for some $s \in \mathbb{R}$. Then there exists an embedding of the coefficient a into $\mathcal{G}([0, T])$, such that the Cauchy problem (4.3) has a unique solution $u \in \mathcal{G}\left([0, T] ; H_{(s)}^{-\infty}\right)$.

Proof. Case II.1. Let us show that by embedding coefficients in the corresponding Colombeau algebras the Cauchy problem has a unique solution $u \in \mathcal{G}\left([0, T] ; H_{\mathcal{L}^{s}}^{s}\right.$. Assume now that the Cauchy problem has another solution $v \in \mathcal{G}\left([0, T] ; H_{\mathcal{L}}^{s}\right)$. At the level of representatives this means

$$
\left\{\begin{aligned}
\partial_{t}^{2}\left(u_{\varepsilon}-v_{\varepsilon}\right)(t)+a_{\varepsilon}(t) \mathcal{L}\left(u_{\varepsilon}-v_{\varepsilon}\right)(t) & =f_{\varepsilon}(t), \\
\left(u_{\varepsilon}-v_{\varepsilon}\right)(0) & =0 \\
\left(\partial_{t} u_{\varepsilon}-\partial_{t} v_{\varepsilon}\right)(0) & =0
\end{aligned}\right.
$$


where $f_{\varepsilon}$ is $C^{\infty}\left([0, T] ; H_{\mathcal{L}}^{s}\right)$-negligible. The corresponding first order system is

$$
\partial_{t}\left(\begin{array}{l}
W_{1, \varepsilon} \\
W_{2, \varepsilon}
\end{array}\right)=\left(\begin{array}{cc}
0 & i \mathcal{L}^{1 / 2} \\
i a_{\varepsilon}(t) \mathcal{L}^{1 / 2} & 0
\end{array}\right)\left(\begin{array}{l}
W_{1, \varepsilon} \\
W_{2, \varepsilon}
\end{array}\right)+\left(\begin{array}{c}
0 \\
f_{\varepsilon}
\end{array}\right),
$$

where $W_{1, \varepsilon}$ and $W_{2, \varepsilon}$ are obtained via the transformation

$$
W_{1, \varepsilon}=\mathcal{L}^{1 / 2}\left(u_{\varepsilon}-v_{\varepsilon}\right), \quad W_{2, \varepsilon}=\partial_{t}\left(u_{\varepsilon}-v_{\varepsilon}\right) .
$$

This system will be studied after $\mathcal{L}$-Fourier transform, as a system of the type

$$
\partial_{t} V_{\varepsilon}(t, \xi)=i \nu(\xi) A_{\varepsilon}(t, \xi) V_{\varepsilon}(t, \xi)+F_{\varepsilon}(t, \xi),
$$

with

$$
F_{\varepsilon}=\left(\begin{array}{c}
0 \\
\mathcal{F}_{\mathcal{L} f_{\varepsilon}}
\end{array}\right)
$$

and

$$
A_{\varepsilon}(t, \xi)=\left(\begin{array}{cc}
0 & 1 \\
a_{\varepsilon}(t) & 0
\end{array}\right)
$$

with Cauchy data

$$
V_{\varepsilon}(0, \xi)=\left(\begin{array}{l}
0 \\
0
\end{array}\right)
$$

For the symmetriser

$$
S_{\varepsilon}(t, \xi)=\left(\begin{array}{cc}
a_{\varepsilon}(t) & 0 \\
0 & 1
\end{array}\right)
$$

define the energy

$$
E_{\varepsilon}(t, \xi):=\left(S_{\varepsilon}(t, \xi) V_{\varepsilon}(t, \xi), V_{\varepsilon}(t, \xi)\right)
$$

We get

$$
\begin{aligned}
\partial_{t} E_{\varepsilon}(t, \xi)= & \left(\partial_{t} S_{\varepsilon}(t, \xi) V_{\varepsilon}(t, \xi), V_{\varepsilon}(t, \xi)\right)+\left(S_{\varepsilon}(t, \xi) \partial_{t} V_{\varepsilon}(t, \xi), V_{\varepsilon}(t, \xi)\right) \\
& +\left(S_{\varepsilon}(t, \xi) V_{\varepsilon}(t, \xi), \partial_{t} V_{\varepsilon}(t, \xi)\right) \\
= & \left(\partial_{t} S_{\varepsilon}(t, \xi) V_{\varepsilon}(t, \xi), V_{\varepsilon}(t, \xi)\right) \\
& +i \nu(\xi)\left(S_{\varepsilon}(t, \xi) A_{\varepsilon}(t, \xi) V_{\varepsilon}(t, \xi), V_{\varepsilon}(t, \xi)\right) \\
& -i \nu(\xi)\left(S_{\varepsilon}(t, \xi) V_{\varepsilon}(t, \xi), A_{\varepsilon}(t, \xi) V_{\varepsilon}(t, \xi)\right) \\
& +\left(S_{\varepsilon}(t, \xi) F_{\varepsilon}(t, \xi), V_{\varepsilon}(t, \xi)\right)+\left(S_{\varepsilon}(t, \xi) V_{\varepsilon}(t, \xi), F_{\varepsilon}(t, \xi)\right) \\
= & \left(\partial_{t} S_{\varepsilon}(t, \xi) V_{\varepsilon}(t, \xi), V_{\varepsilon}(t, \xi)\right) \\
& +i \nu(\xi)\left(\left(S_{\varepsilon} A_{\varepsilon}-A_{\varepsilon}^{*} S_{\varepsilon}\right)(t, \xi) V_{\varepsilon}(t, \xi), V_{\varepsilon}(t, \xi)\right) \\
& +\left(S_{\varepsilon}(t, \xi) F_{\varepsilon}(t, \xi), V_{\varepsilon}(t, \xi)\right)+\left(V_{\varepsilon}(t, \xi), S_{\varepsilon}(t, \xi) F_{\varepsilon}(t, \xi)\right) \\
= & \left(\partial_{t} S_{\varepsilon}(t, \xi) V_{\varepsilon}(t, \xi), V_{\varepsilon}(t, \xi)\right)+2 \operatorname{Re}\left(S_{\varepsilon}(t, \xi) F_{\varepsilon}(t, \xi), V_{\varepsilon}(t, \xi)\right) \\
\leq & \left\|\partial_{t} S_{\varepsilon}\right\|\left|V_{\varepsilon}(t, \xi)\right|^{2}+2 \operatorname{Re}\left(S_{\varepsilon}(t, \xi) F_{\varepsilon}(t, \xi), V_{\varepsilon}(t, \xi)\right) \\
\leq & \left\|\partial_{t} S_{\varepsilon}\right\|\left|V_{\varepsilon}(t, \xi)\right|^{2}+2\left\|S_{\varepsilon}\right\|\left|F_{\varepsilon}(t, \xi) \| V_{\varepsilon}(t, \xi)\right| .
\end{aligned}
$$


Assuming for the moment that $\left|V_{\varepsilon}(t, \xi)\right|>1$, we get the energy estimate

$$
\begin{aligned}
\partial_{t} E_{\varepsilon}(t, \xi) & \leq\left\|\partial_{t} S_{\varepsilon}\right\|\left|V_{\varepsilon}(t, \xi)\right|^{2}+2\left\|S_{\varepsilon}\right\|\left|F_{\varepsilon}(t, \xi) \| V_{\varepsilon}(t, \xi)\right| \\
& \leq\left(\left\|\partial_{t} S_{\varepsilon}\right\|+2\left\|S_{\varepsilon}\right\|\left|F_{\varepsilon}(t, \xi)\right|\right)\left|V_{\varepsilon}(t, \xi)\right|^{2} \\
& \leq\left(\left|\partial_{t} a_{\varepsilon}(t)\right|+\left|a_{\varepsilon}(t) \| F_{\varepsilon}(t, \xi)\right|\right)\left|V_{\varepsilon}(t, \xi)\right|^{2} \\
& \leq c \omega(\varepsilon)^{-L-1} E_{\varepsilon}(t, \xi),
\end{aligned}
$$

i.e. we obtain

$$
\partial_{t} E_{\varepsilon}(t, \xi) \leq c \omega(\varepsilon)^{-L-1} E_{\varepsilon}(t, \xi),
$$

for some constant $c>0$. By Gronwall's lemma applied to inequality (7.8) we conclude that for all $T>0$

$$
E_{\varepsilon}(t, \xi) \leq \exp \left(c \omega(\varepsilon)^{-L-1} T\right) E_{\varepsilon}(0, \xi) .
$$

Hence, inequalities (6.8) yield

$$
\begin{aligned}
c_{0}\left|V_{\varepsilon}(t, \xi)\right|^{2} \leq E_{\varepsilon}(t, \xi) & \leq \exp \left(c \omega(\varepsilon)^{-L-1} T\right) E_{\varepsilon}(0, \xi) \\
& \leq \exp \left(c_{1} \omega(\varepsilon)^{-L-1} T\right)\left|V_{\varepsilon}(0, \xi)\right|^{2},
\end{aligned}
$$

for the constant $c_{1}$ independent of $t \in[0, T]$ and $\xi$.

By putting $\omega^{-L-1}(\varepsilon) \sim \log \varepsilon$, we get

$$
\left|V_{\varepsilon}(t, \xi)\right|^{2} \leq c \varepsilon^{-L-1}\left|V_{\varepsilon}(0, \xi)\right|^{2}
$$

for some constant $c$ and some (new) $L$. Since $\left|V_{\varepsilon}(0, \xi)\right|=0$, we have

$$
\left|V_{\varepsilon}(t, \xi)\right| \equiv 0
$$

for all $\xi$ and for $t \in[0, T]$.

Now consider the case when $\left|V_{\varepsilon}(t, \xi)\right|<1$. Assume that $\left|V_{\varepsilon}(t, \xi)\right| \geq c \omega(\varepsilon)^{\alpha}$ for some constant $c$ and $\alpha>0$. It means

$$
\frac{1}{\left|V_{\varepsilon}(t, \xi)\right|} \leq C \omega(\varepsilon)^{-\alpha} \text {. }
$$

Then the estimate for the energy becomes

$$
\partial_{t} E_{\varepsilon}(t, \xi) \leq C \omega(\varepsilon)^{-L_{1}} E_{\varepsilon}(t, \xi),
$$

where $L_{1}=L+\max \{1, \alpha\}$, and by Gronwall's lemma

$$
\left|V_{\varepsilon}(t, \xi)\right|^{2} \leq \exp \left(C^{\prime} \omega(\varepsilon)^{-L_{1}} T\right)\left|V_{\varepsilon}(0, \xi)\right|^{2} \text {. }
$$

And again, by putting $\omega^{-L_{1}}(\varepsilon) \sim \log \varepsilon$, we get

$$
\left|V_{\varepsilon}(t, \xi)\right|^{2} \leq c^{\prime} \varepsilon^{-L_{1}}\left|V_{\varepsilon}(0, \xi)\right|^{2}
$$

for some $c^{\prime}$ and some (new) $L_{1}$. Since $\left|V_{\varepsilon}(0, \xi)\right|=0$, we have

$$
\left|V_{\varepsilon}(t, \xi)\right| \equiv 0
$$

for all $t \in[0, T]$ and $\xi$. 
The last case is when $\left|V_{\varepsilon}(t, \xi)\right| \leq c \omega(\varepsilon)^{\alpha}$ for some constant $c$ and $\alpha>0$. Thus, the first part is proved.

Case II.2. Here we will repeat some discussions of the first part but we will also use the quasi-symmetrisers. Now, let us show that by embedding coefficients in the corresponding Colombeau algebras the Cauchy problem has a unique solution $u \in \mathcal{G}\left([0, T] ; H_{(s)}^{-\infty}\right)$. Assume now that the Cauchy problem has another solution $v \in \mathcal{G}\left([0, T] ; H_{(s)}^{-\infty}\right)$. At the level of representatives this means that

$$
\left\{\begin{aligned}
\partial_{t}^{2}\left(u_{\varepsilon}-v_{\varepsilon}\right)(t)+a_{\varepsilon}(t) \mathcal{L}\left(u_{\varepsilon}-v_{\varepsilon}\right)(t) & =f_{\varepsilon}(t), \\
\left(u_{\varepsilon}-v_{\varepsilon}\right)(0) & =0 \\
\left(\partial_{t} u_{\varepsilon}-\partial_{t} v_{\varepsilon}\right)(0) & =0
\end{aligned}\right.
$$

where $f_{\varepsilon}$ is $C^{\infty}\left([0, T] ; H_{(s)}^{-\infty}\right)$-negligible. The corresponding first order system is

$$
\partial_{t}\left(\begin{array}{l}
W_{1, \varepsilon} \\
W_{2, \varepsilon}
\end{array}\right)=\left(\begin{array}{cc}
0 & i \mathcal{L}^{1 / 2} \\
i a_{\varepsilon}(t) \mathcal{L}^{1 / 2} & 0
\end{array}\right)\left(\begin{array}{l}
W_{1, \varepsilon} \\
W_{2, \varepsilon}
\end{array}\right)+\left(\begin{array}{c}
0 \\
f_{\varepsilon}
\end{array}\right),
$$

where $W_{1, \varepsilon}$ and $W_{2, \varepsilon}$ are obtained via the transformation

$$
W_{1, \varepsilon}=\mathcal{L}^{1 / 2}\left(u_{\varepsilon}-v_{\varepsilon}\right), \quad W_{2, \varepsilon}=\partial_{t}\left(u_{\varepsilon}-v_{\varepsilon}\right) .
$$

This system will be studied after $\mathcal{L}$-Fourier transform, as a system of the type

$$
\partial_{t} V_{\varepsilon}(t, \xi)=i v(\xi) A_{\varepsilon}(t, \xi) V_{\varepsilon}(t, \xi)+F_{\varepsilon}(t, \xi),
$$

with

$$
F_{\varepsilon}=\left(\begin{array}{c}
0 \\
\mathcal{F}_{\mathcal{L}} f_{\varepsilon}
\end{array}\right)
$$

and

$$
A_{\varepsilon}(t, \xi)=\left(\begin{array}{cc}
0 & 1 \\
a_{\varepsilon}(t) & 0
\end{array}\right)
$$

with Cauchy data

$$
V_{\varepsilon}(0, \xi)=\left(\begin{array}{l}
0 \\
0
\end{array}\right)
$$

For the quasi-symmetriser $Q_{\varepsilon}(t, \delta)$, defined as

$$
Q_{\varepsilon}(t, \delta):=\left(\begin{array}{cc}
a_{\varepsilon}(t) & 0 \\
0 & 1
\end{array}\right)+\delta^{2}\left(\begin{array}{ll}
1 & 0 \\
0 & 0
\end{array}\right),
$$

we define the energy

$$
E_{\varepsilon}(t, \xi, \delta)=\left(Q_{\varepsilon}(t, \delta) V(t, \xi), V(t, \xi)\right) .
$$


By direct computations we get

$$
\begin{gathered}
\partial_{t} E_{\varepsilon}(t, \xi, \delta)=\left(\partial_{t} Q_{\varepsilon}(t, \delta) V(t, \xi), V(t, \xi)\right)+i v(\xi)\left(\left(Q_{\varepsilon} A-A^{*} Q_{\varepsilon}\right)(t) V, V\right) \\
+2 \operatorname{Re}\left(Q_{\varepsilon}(t, \delta) F_{\varepsilon}(t, \xi), V_{\varepsilon}(t, \xi)\right) .
\end{gathered}
$$

By using properties that were established in the proof of Theorem 2.3 and continuing to discuss as in the first part, from the last equality we conclude that the Cauchy problem (4.3) has a unique solution $u \in \mathcal{G}\left([0, T] ; H_{(s)}^{-\infty}\right)$ for all $s \in \mathbb{R}$.

It completes the proof of Theorem 7.2.

Proof of Theorem 4.4. Case II.1. We now want to compare the classical solution $\tilde{u}$ given by Theorem 2.1 with the very weak solution $u$ provided by Theorem 4.4. By the definition of the classical solution we know that

$$
\left\{\begin{aligned}
\partial_{t}^{2} \widetilde{u}(t)+a(t) \mathcal{L} \widetilde{u}(t) & =f(t), \\
\widetilde{u}(0) & =u_{0} \in \mathcal{H}, \\
\partial_{t} \tilde{u}(0) & =u_{1} \in \mathcal{H} .
\end{aligned}\right.
$$

By the definition of the very weak solution $u$, there exists a representative $\left(u_{\varepsilon}\right)_{\varepsilon}$ of $u$ such that

$$
\left\{\begin{aligned}
\partial_{t}^{2} u_{\varepsilon}(t)+a_{\varepsilon}(t) \mathcal{L} u_{\varepsilon}(t) & =f_{\varepsilon}(t), \\
u_{\varepsilon}(0) & =u_{0} \in \mathcal{H}, \\
\partial_{t} u_{\varepsilon}(0) & =u_{1} \in \mathcal{H},
\end{aligned}\right.
$$

for suitable embeddings of the coefficient $a$ and of the source term $f$. Noting that for $a \in L_{1}^{\infty}([0, T])$ the nets $\left(a_{\varepsilon}-a\right)_{\varepsilon}$ is converging to 0 in $C([0, T])$, we can rewrite (7.9) as

$$
\left\{\begin{aligned}
\partial_{t}^{2} \widetilde{u}(t)+\widetilde{a}_{\varepsilon}(t) \mathcal{L} \widetilde{u}(t) & =\widetilde{f}_{\varepsilon}(t), \\
\widetilde{u}(0) & =u_{0} \in \mathcal{H}, \\
\partial_{t} \widetilde{u}(0) & =u_{1} \in \mathcal{H},
\end{aligned}\right.
$$

where $\widetilde{f}_{\varepsilon} \in C\left([0, T] ; H_{\mathcal{L}}^{s}\right)$ and $\widetilde{a}_{\varepsilon}$ are other representations of $f$ and $a$. From (7.10) and (7.11) we get that $\tilde{u}-u_{\varepsilon}$ solves the Cauchy problem

$$
\left\{\begin{aligned}
\partial_{t}^{2}\left(\tilde{u}-u_{\varepsilon}\right)(t)+a_{\varepsilon}(t) \mathcal{L}\left(\tilde{u}-u_{\varepsilon}\right)(t) & =n_{\varepsilon}(t), \\
\left(\tilde{u}-u_{\varepsilon}\right)(0) & =0, \\
\left(\partial_{t} \tilde{u}-\partial_{t} u_{\varepsilon}\right)(0) & =0 .
\end{aligned}\right.
$$

As in the first part of the proof we arrive, after reduction to a system and by application of the Fourier transform to estimate $\left|\left(\widetilde{V}-V_{\varepsilon}\right)(t, \xi)\right|$ in terms of $(\widetilde{V}-$ $\left.V_{\varepsilon}\right)(0, \xi)$ and the right-hand side $n_{\varepsilon}(t)$, to the energy estimate

$$
\begin{aligned}
\partial_{t} E_{\varepsilon}(t, \xi) \leq & \left|\partial_{t} a_{\varepsilon}(t)\right|\left|\left(\tilde{V}-V_{\varepsilon}\right)(t, \xi)\right|^{2} \\
& +2\left|a_{\varepsilon}(t)\right|\left|n_{\varepsilon}(t, \xi)\right|\left|\left(\widetilde{V}-V_{\varepsilon}\right)(t, \xi)\right| .
\end{aligned}
$$

Since the coefficients are regular enough, we simply get

$$
\partial_{t} E_{\varepsilon}(t, \xi) \leq c_{1}\left|\left(\widetilde{V}-V_{\varepsilon}\right)(t, \xi)\right|^{2}+c_{2}\left|n_{\varepsilon}(t, \xi)\right|\left|\left(\widetilde{V}-V_{\varepsilon}\right)(t, \xi)\right| .
$$


Since $\left(\widetilde{V}-V_{\varepsilon}\right)(0, \xi)=0$ and $n_{\varepsilon} \rightarrow 0$ in $C\left([0, T] ; H_{\mathcal{L}}^{s}\right)$ and continue to discussing as in Theorem 7.2 we conclude that

$$
\left|\left(\widetilde{V}-V_{\varepsilon}\right)(t, \xi)\right| \leq c \omega(\varepsilon)^{\alpha}
$$

for some constant $c$ and $\alpha>0$. Then we have $u_{\varepsilon} \rightarrow \tilde{u}$ in $C\left([0, T] ; H_{\mathcal{L}}^{1+s}\right) \cap$ $C^{1}\left([0, T] ; H_{\mathcal{L}}^{s}\right)$. Moreover, since any other representative of $u$ will differ from $\left(u_{\varepsilon}\right)_{\varepsilon}$ by a $C^{\infty}\left([0, T] ; H_{\mathcal{L}}^{s}\right)$-negligible net, the limit is the same for any representative of $u$.

Case II.2. This part can be proven as the previous Case II.1 with slight modifications.

Open Access This article is distributed under the terms of the Creative Commons Attribution 4.0 International License (http://creativecommons.org/licenses/by/4.0/), which permits unrestricted use, distribution, and reproduction in any medium, provided you give appropriate credit to the original author(s) and the source, provide a link to the Creative Commons license, and indicate if changes were made.

\section{References}

1. Abreu, L.D.: Sampling and interpolation in Bargmann-Fock spaces of polyanalytic functions. Appl. Comput. Harmon. Anal., 29, 287-302, 2010

2. Abreu, L.D., Balazs, P., De Gosson, M., Mouayn, Z.: Discrete coherent states for higher Landau levels. Ann. Phys., 363, 337-353, 2015

3. Ali, S.T., Bagarello, F., Gazeau, J.P.: Quantizations from reproducing kernel spaces. Ann. Phys., 332, 127-142, 2012

4. BARI, N.K.: Biorthogonal systems and bases in Hilbert space. Moskov. Gos. Univ. Učenye Zapiski Matematika, 148(4), 69-107, 1951.

5. Bergeron, H., Gazeau, J.P.: Integral quantizations with two basic examples. Ann. Phys., 344, 43-68, 2014

6. Bonfiglioli, A., Lanconelli, E., Uguzzoni, F.: Stratified Lie groups and potential theory for their sub-Laplacians. Springer Monographs in Mathematics. Springer, Berlin, (2007)

7. BRonšTen, M.D.: The Cauchy problem for hyperbolic operators with characteristics of variable multiplicity. Trudy Moskov. Mat. Obshch., 41, 83-99, 1980

8. Bruno, T., Calzi, M.: Weighted sub-Laplacians on Métivier groups: essential selfadjointness and spectrum. Proc. Am. Math. Soc., 145, 3579-3594, 2017

9. Cotfas, N., Gazeau, J.P., Grorska, K.: Complex and real Hermite polynomials and related quantizations. J. Phys. A: Math. Theor., 43(30): 305304, 1-14, 2010

10. Cicognani, M., Colombini, F.: A well-posed Cauchy problem for an evolution equation with coefficients of low regularity. J. Differ. Equ., 254(8), 3573-3595, 2013

11. Colombini, F., De Giorgi, E., Spagnolo, S.: Sur les équations hyperboliques avec des coefficients qui ne dépendent que du temps. Ann. Scuola Norm. Sup. Pisa Cl. Sci. (4), 6(3):511-559, 1979

12. Colombini, F., Del Santo, D., Reissig, M.: On the optimal regularity of coefficients in hyperbolic Cauchy problems. Bull. Sci. Math., 127(4). 328-347, 2003

13. Colombini, F., Jannelli, E., Spagnolo, S.: Nonuniqueness in hyperbolic Cauchy problems. Ann. Math. (2), 126(3), 495-524, 1987 
14. Colombini, F., Kinoshita, T.: On the Gevrey well posedness of the Cauchy problem for weakly hyperbolic equations of higher order. J. Differ. Equ., 186(2), 394-419, 2002

15. Colombini, F., Spagnolo, S.: An example of a weakly hyperbolic Cauchy problem not well posed in $C^{\infty}$. Acta Math., 148, 243-253, 1982

16. D'AnCona, P., Spagnolo, S.: Quasi-symmetrization of hyperbolic systems and propagation of the analytic regularity. Boll. Unione Mat. Ital. Sez. B Artic. Ric. Mat. (8), 1(1), 169-185, 1998

17. Dasgupta, A., Ruzhansky, M.: Gevrey functions and ultradistributions on compact Lie groups and homogeneous spaces. Bull. Sci. Math., 138(6), 756-782, 2014

18. Dasgupta, A., Ruzhansky, M.: Eigenfunction expansions of ultradifferentiable functions and ultradistributions. Trans. Am. Math. Soc., 368(12), 8481-8498, (2016)

19. Delgado, J., Ruzhansky, M., Tokmagambetov, N.: Schatten classes, nuclearity and nonharmonic analysis on compact manifolds with boundary. J. Math. Pures Appl., 107(6), 758-783, 2017

20. Fischer, V., Ruzhansky, M., Taranto, C.: On sub-Laplacian gevrey spaces. preprint.

21. Fock, V.: Bemerkung zur Quantelung des harmonischen Oszillators im Magnetfeld. $Z$. Phys. A, 47(5-6): 446-448, 1928

22. Garetto, C., Ruzhansky, M.: On the well-posedness of weakly hyperbolic equations with time-dependent coefficients. J. Differ. Equ., 253(5), 1317-1340, 2012

23. Garetto, C., Ruzhansky, M.: Weakly hyperbolic equations with non-analytic coefficients and lower order terms. Math. Ann., 357(2), 401-440, 2013

24. Garetto, C., Ruzhansky, M.: Wave equation for sums of squares on compact Lie groups. J. Differ. Equ., 258, 4324-4347, 2015

25. Garetto, C., Ruzhansky, M.: Hyperbolic second order equations with non-regular time dependent coefficients. Arch. Rational Mech. Anal., 217(1), 113-154, 2015

26. Gelfand, I.M.: Some questions of analysis and differential equations. Am. Math. Soc. Transl. (2), 26, 201-219, 1963

27. Gosson DE, M.: Spectral properties of a class of generalized Landau operators. Comm. Partial Differ. Equ., 33(11), 2096-2104, 2008

28. HelfFer, B., Robert, D.: Asymptotique des niveaux d'énergie pour des hamiltoniens à un degr é de liberté. Duke Math. J., 49(4), 853-868, 1982

29. HörmanN, G., DE Hoop, M.V.: Microlocal analysis and global solutions of some hyperbolic equations with discontinuous coefficients. Acta Appl. Math., 67(2), 173-224, 2001

30. HörmanN, G., DE Hoop, M.V.: Detection of wave front set perturbations via correlation: foundation for wave-equation tomography. Appl. Anal., 81(6), 1443-1465, 2002

31. Hurd, A.E., SATtinger, D.H.: Questions of existence and uniqueness for hyperbolic equations with discontinuous coefficients. Trans. Am. Math. Soc., 132, 159-174, 1968

32. Haimi, A., Hedenmalm, H.: The polyanalytic Ginibre ensembles. J. Stat. Phys., 153(1), $10-47,2013$

33. IngLis, J.: Spectral inequalities for operators on H-type groups. J. Spectr. Theory, 2 , 79-105, 2012

34. Ismail, M.: Analytic properties of complex Hermite polynomials. Trans. Am. Math. Soc., 368(2), 1189-1210, 2016

35. Kawamoto, M.: Exponential decay property for eigenfunctions of Landau-Stark Hamiltonia. Rep. Math. Phys., 77(1), 129-140, 2016

36. Kinoshita, T., Spagnolo, S.: Hyperbolic equations with non-analytic coefficients. Math. Ann., 336, 551-569, 2006

37. Korotyaev, E., Pushnitski, A.: A trace formula and high-energy spectral asymptotics for the perturbed Landau Hamiltonian. J. Funct. Anal., 217, 221-248, 2004

38. Kinoshita, T., Spagnolo, S.: Hyperbolic equations with non-analytic coefficients. Math. Ann., 336(3), 551-569, 2006

39. Landau, L.: Diamagnetismus der Metalle. Z. Phys. A, 64(9-10): 629-637, 1930

40. Lungenstrass, T., Raikov, G.: A trace formula for long-range perturbations of the Landau Hamiltonian. Ann. Henri Poincare, 15, 1523-1548, 2014 
41. Matsumoto, H.: Classical and non-classical eigenvalue asymptotics for magnetic Schrödinger operators. J. Funct. Anal., 95, 460-482, 1991

42. Namark, M.A.: Linear differential operators. Part II: Linear differential operators in Hilbert space. With additional material by the author, and a supplement by V. È. Ljance. Translated from the Russian by E. R. Dawson. English translation edited by W. N. Everitt. Frederick Ungar Publishing Co., New York, 1968

43. Nicola, F., Rodino, L.: Global pseudo-differential calculus on Euclidean spaces, volume 4 of Pseudo-Differ. Oper. Theory Appl. Birkhäuser Verlag, Basel, 2010

44. Nakamura, Sh.: Gaussian decay estimates for the eigenfunctions of magnetic Schrödinger operators. Commun. Partial Differ. Equ., 21(5-6), 993-1006, 1996

45. Oberguggenberger, M.: Multiplication of distributions and applications to partial differential equations, volume 259 of Pitman Research Notes in Mathematics Series. Longman Scientific \& Technical, Harlow, 1992

46. Perelomov, A.: Generalized Coherent States and Their Applications. Texts and Monographs in Physics. Springer, Berlin, 1986

47. Persson, M.: Eigenvalue asymptotics of the even-dimensional exterior LandauNeumann Hamiltonian. Adv. Math. Phys., Article ID 873704, 2009

48. Pushnitski, A., Raikov, G., Villegas-Blas, C.: Asymptotic density of eigenvalue clusters for the perturbed Landau Hamiltonian. Comm. Math. Phys., 320, 425-453, 2013

49. Pushnitski, A., Rozenblum, G.: Eigenvalue clusters of the Landau Hamiltonian in the exterior of a compact domain. Doc. Math., 12, 569-586, 2007

50. Rozenblum, G., TAshchiyan, G.: On the spectral properties of the perturbed Landau Hamiltonian. Comm. Partial Differ. Equ., 33, 1048-1081, 2008

51. Ruzhansky, M., Turunen, V.: Pseudo-differential operators and symmetries. Background analysis and advanced topics, volume 2 of Pseudo-Differential Operators. Theory and Applications. Birkhäuser Verlag, Basel, 2010

52. RuZhansky, M., Turunen, V.: Global quantization of pseudo-differential operators on compact Lie groups, $\mathrm{SU}(2), 3$-sphere, and homogeneous spaces. Int. Math. Res. Not. IMRN, (11), 2439-2496, 2013

53. RuZhansky, M., Tokmagambetov, N.: Nonharmonic analysis of boundary value problems. Int. Math. Res. Not. IMRN, (12), 3548-3615, 2016

54. Ruzhansky, M., Tokmagambetov, N.: Very weak solutions of wave equation for Landau Hamiltonian with irregular electromagnetic field. Lett. Math. Phys. 107, 591618, 2017

55. Ruzhansky, M., Tokmagambetov, N.: Nonharmonic analysis of boundary value problems without WZ condition. Math. Model. Nat. Phenom. 12, 115-140, 2017. (arXiv:1610.02159)

56. Ruzhansky, M., Taranto, C.: Time-dependent wave equations on graded groups. arXiv: 1705.03047

57. Sambou, D.: Lieb-Thirring type inequalities for non-self-adjoint perturbations of magnetic Schrödinger operators. J. Funct. Anal., 266, 5016-5044, 2014

58. Schwartz, L.: Sur l'impossibilité de la multiplication des distributions. C. R. Acad. Sci. Paris, 239, 847-848, 1954

59. ShKalikov, A.A.: Basis property of eigenfunctions of ordinary differential operators with integral boundary conditions. Vestnik Moskov. Univ. Ser. I Mat. Mekh., 120(6), 12-21, 1982 


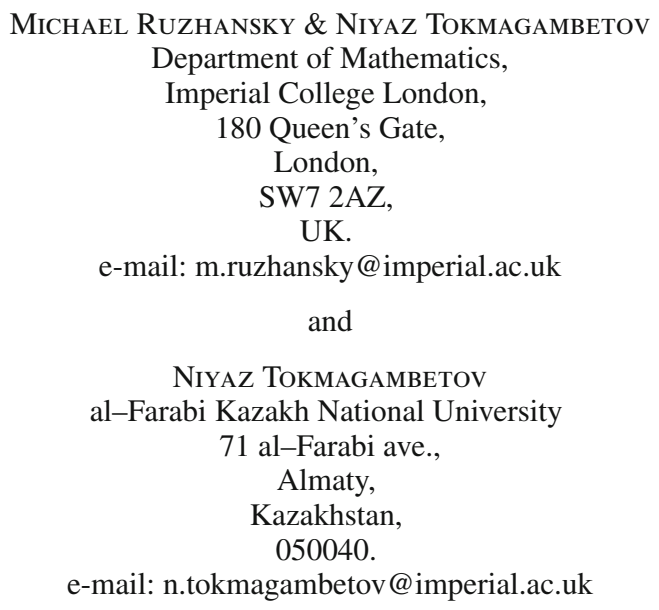

(Received November 8, 2016 / Accepted July 13, 2017)

Published online July 26, 2017 - (C) The Author(s) (2017)

This article is an open access publication 